\title{
Sulfur-Promoted DABCO-Catalyzed Oxidative Trimerization of Phenylacetonitriles
}

\author{
Thanh Binh Nguyen,* and Pascal Retailleau
}

Institut de Chimie des Substances Naturelles, CNRS UPR 2301, Université Paris-Sud, Université Paris-Saclay, 1 avenue de la Terrasse, 91198 Gif-sur-Yvette, France

thanh-binh.nguyen@cnrs.fr 
Crystallographic data collection, structure determination and refinement

Procedure for large scale synthesis of 2 a

SI9

Copies of ${ }^{1} \mathrm{H}$ and ${ }^{13} \mathrm{C}$ NMR spectra

SI10 


\section{Crystallographic data collection, structure determination and refinement}

Yellow crystals were obtained for the 2-(5-amino-3,4-diphenyl-2H-pyrrol-2-yl)-2-phenylacetonitrile compound, 2a, and three other substituted phenyl derivatives, $2 \mathbf{e}, \mathbf{2 c}$ and $\mathbf{2} \mathbf{c}$, the two latter being two isomers, from slow evaporation of dichloromethane as crystallization solvent. The crystalline needles of the first compound were tested for x-ray diffraction at room temperature using a RIGAKU diffractometer constituted by a MM007 HF rotating-anode generator, delivering copper radiation through Osmic CMF confocal optics, and a Rapid II curved Image Plate detector. Fs process ${ }^{1}$ software under the CrystalClear $2.0^{2}$ suite was used to integrate and scale the data, applying multiscan $R E Q A B^{1}$ for the absorption correction. The structure determination for the three other compounds were performed using a RIGAKU XtaLabPro diffractometer equipped with a Mo microfocus sealed tube generator coupled to a double-bounce confocal Max-Flux ${ }^{\circledR}$ multilayer optic and a HPAD PILATUS3 R 200K detector. Data collections were also carried out at room temperature using the data processing software, CrysAlisPro. ${ }^{3}$ All the structures were solved by intrinsic phasing methods (SHELXT program $)^{4}$ and refined by full-matrix least-squares methods on $F^{2}$ using SHELX-L. ${ }^{5}$ All nonhydrogen atoms of the molecules of interest improved by anisotropic refinement. Most of the $\mathrm{H}$ atoms were identified in difference maps. Methyl $\mathrm{H}$ atoms in $\mathbf{2 e}, \mathbf{2 c}$, and $\mathbf{2} \mathbf{c}$ ' were idealized and included as rigid groups allowed to rotate but not tip and refined with $U_{\text {iso }}$ set to $1.5 U_{\text {eq }}(\mathrm{C})$ of the parent carbon atom. All other $\mathrm{H}$ atoms bound to carbon atoms were positioned geometrically and refined with $U_{\text {iso }}$ set to $1.2 U_{\text {eq }}(C)$ of the parent carbon atom. The amine $\mathrm{H}$ atoms were also found in a difference Fourier map and their positions were refined with bond length restraints (DFIX, SADI instructions) and with relative displacement coefficients set at $1.2 U_{\mathrm{cq}}(\mathrm{N}$ atom). The methoxy-phenyl group attached to the acetonitrile moiety in $\mathbf{2 e}$ appeared disordered over two orthogonal sites refined with equivalent $(0.5)$ occupancy factors. Large dynamic disorder observed for one phenyl group in 2a and the three

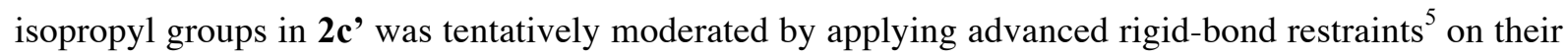
anisotropic displacement parameters (RIGU instructions). $2 c$ was a weak, twinned diffractor, and after reduction and correction for absorption, Lorentz and polarization effects, 7194 independent reflections were retained $(\mathrm{R}(\mathrm{int})=0.161)$ up to a resolution sphere of $\sin (\theta) / \lambda=0.56 \AA^{-}$. The structure was solved in the triclinic space group. The pseudo-merohedral twining was confirmed by the TwinRotMat function in PLATON, ${ }^{6}$ and the twin law (-1 00 / 0 - 10 / 0.217 -0.971 1) was detected. An HKLF5 file was generated with a refined minor twin fraction of $0.335(5)$. The structure was then refined upon 339 parameters against 4031 reflections of the first domain, applying the following weighted refinement scheme: $w=1 /\left[\sigma^{2}\left(\mathrm{~F}_{\circ}^{2}\right)+(0.0477 \mathrm{P})^{2}+1.245 \mathrm{P}\right]$ with $\mathrm{P}=\left[\max \left(\mathrm{F}_{\circ}^{2}, 0\right)+2 \mathrm{~F}_{\circ}^{2}\right] / 3$. X-ray crystallography data collection parameters and structure refinement statistics are reported in table 1 . Ortep views are shown in Figure 1.

With respect to the crystal packing, all the molecules are assembled as inversion-related dimer through a pair of $\mathrm{H}$-bonds between one hydrogen of the amine group and the pyrrole $\mathrm{N}$ atom of the partner 
forming a $\mathrm{R}_{2}^{2}(8)$ ring motif. ${ }^{7}$ In the case of $\mathbf{2 a}$, this corresponds to a pseudo-inversion since the compound crystallizes in the Sohncke space group, $P 2$, with two molecules independently refined in the asymmetric unit, with respect to small variations in their own conformation. Regarding the two isomers case, the second amine hydrogen interacts with the Z-phenyl group which is orthogonal

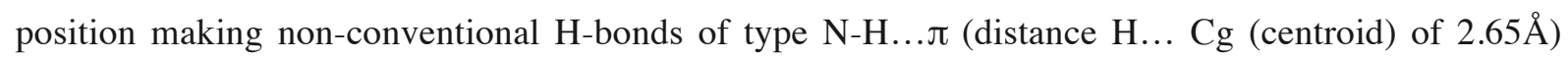
(Figure 1). This interaction occurs is also for 2a (distance $2.95 \AA$ ) but is no longer present in $\mathbf{2 e}$, due to the remote and inclined position of the phenyl. Within $\mathbf{2 c}$ ' packing, the E-isomer brings the acetonitrile $\mathrm{N}$ atom of the inversion-related vicinal molecule nearby the second amine $\mathrm{H}$. The 16 atoms ring motif which is formed encompasses the former one (Figure 2).

CCDC 1883985-1883986 (2a-2e), 1846812 (compound 2c), and 1844000 (compound 2c') contain the supplementary crystallographic data for this paper. These data can be obtained free of charge from The Cambridge Crystallographic Data Centre via www.ccdc.cam.ac.uk/data_request/cif. 

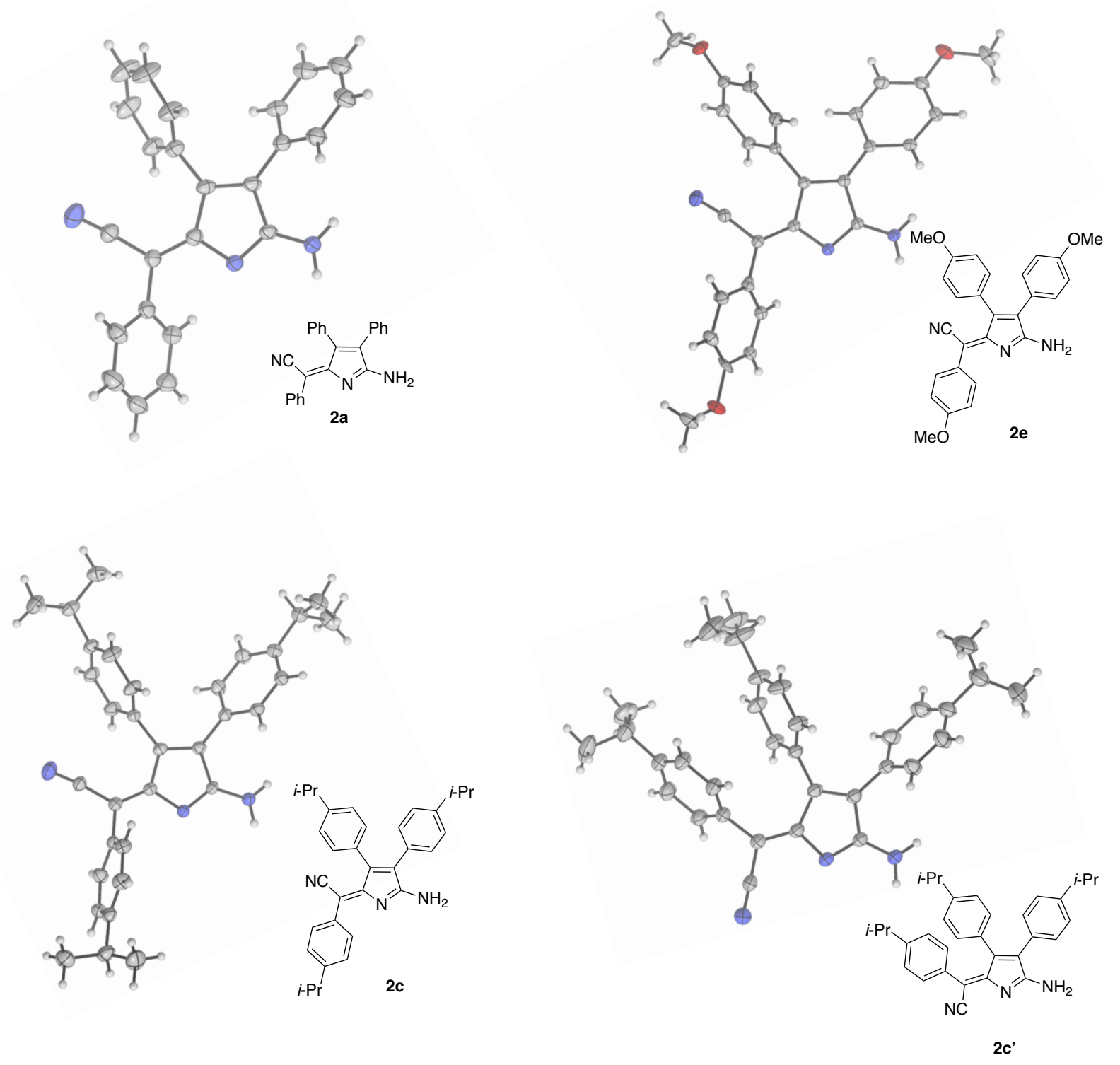

Figure S1 (upleft) Ortep plot of one conformer of 2a (upright) Ortep plot of 2e (disorder is not shown for clarity) (downleft) Ortep plot of 2c (downright) Ortep plot of 2c'. Ellipsoids are drawn at 30\% of probability and hydrogen atoms, with sphere radius of arbitrary size. 

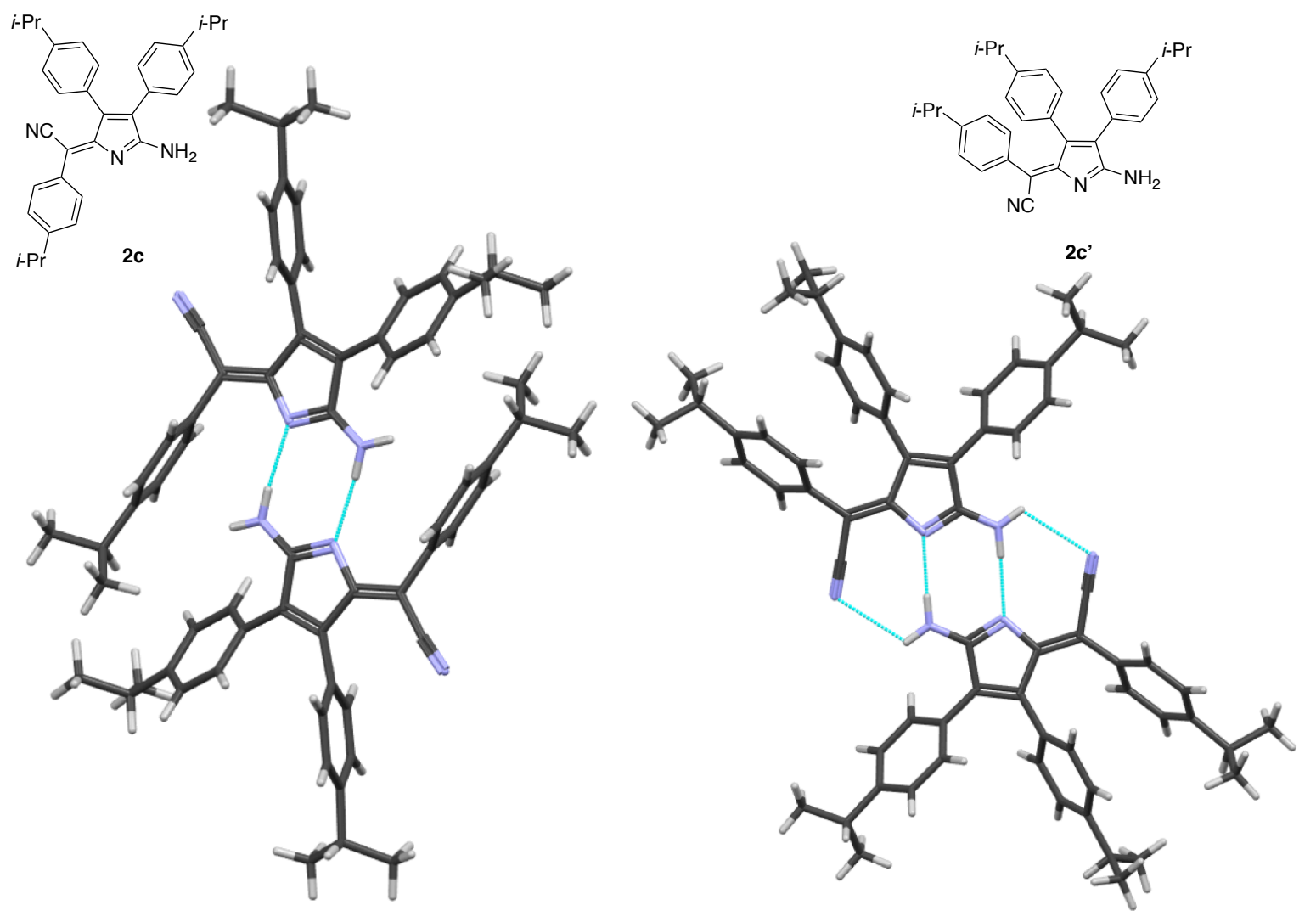

Figure S2 dimeric association through crystallographic center of inversion (left) $2 c$; (right) $2 c$ '. 
Table S1 Crystal data and structure refinement for $2 \mathrm{a}, \mathbf{2 e}, \mathbf{2 c}$ and $2 \mathrm{c}$.

\begin{tabular}{|c|c|c|c|c|c|}
\hline \multicolumn{2}{|l|}{ Identification code } & $2 a$ & $2 e$ & $2 c$ & $2 c^{\prime}$ \\
\hline \multicolumn{2}{|l|}{ Empirical formula } & $\mathrm{C}_{24} \mathrm{H}_{17} \mathrm{~N}_{3}$ & $\mathrm{C}_{27} \mathrm{H}_{23} \mathrm{~N}_{3} \mathrm{O}_{3}$ & $\mathrm{C}_{33} \mathrm{H}_{35} \mathrm{~N}_{3}$ & $\mathrm{C}_{33} \mathrm{H}_{35} \mathrm{~N}_{3}$ \\
\hline \multicolumn{2}{|l|}{ Formula weight } & 347.40 & 437.48 & 473.64 & 473.64 \\
\hline \multicolumn{2}{|l|}{ Temperature (K) } & \multicolumn{4}{|c|}{$293(2)$} \\
\hline \multicolumn{2}{|l|}{ Wavelength $(\AA)$} & 1.54187 & \multicolumn{3}{|c|}{0.71073} \\
\hline \multicolumn{2}{|l|}{$\begin{array}{l}\text { Crystal system, } \\
\text { Space group }\end{array}$} & $\begin{array}{l}\text { Monoclinic, } \\
\quad \text { P } 2_{1}\end{array}$ & $\begin{array}{l}\text { Triclinic, } \\
\text { P -1 }\end{array}$ & $\begin{array}{l}\text { Triclinic, } \\
\text { P -1 }\end{array}$ & $\begin{array}{l}\text { Monoclinic, } \\
\mathrm{C} 2 / \mathrm{c}\end{array}$ \\
\hline $\begin{array}{l}\text { Unit cell dimensions } \\
(\AA, \circ)\end{array}$ & $\begin{array}{l}\mathrm{a} \mid \AA \\
\mathrm{b} \\
\mathrm{c} \\
\left.\alpha\right|^{\circ} \\
\beta \\
\gamma \\
\end{array}$ & $\begin{array}{c}9.2883(7) \\
23.2285(8) \\
9.3154(7) \\
90 \\
109.472(8) \\
90\end{array}$ & $\begin{array}{c}5.9434(6) \\
12.1854(12) \\
15.7437(14) \\
88.834(8) \\
84.137(8) \\
81.962(8)\end{array}$ & $\begin{array}{c}9.4854(7) \\
12.5272(10) \\
13.1934(16) \\
63.411(10) \\
89.962(8) \\
80.155(6)\end{array}$ & $\begin{array}{c}25.855(2) \\
9.4237(6) \\
25.289(2) \\
90 \\
114.109(10) \\
90\end{array}$ \\
\hline \multicolumn{2}{|l|}{ Volume $\left(\AA^{3}\right)$} & $1894.9(2)$ & $1123.07(18)$ & $1376.1(2)$ & $5624.0(8)$ \\
\hline \multicolumn{2}{|c|}{$\begin{array}{l}\mathrm{Z}, \\
\text { Calculated density }\left(\mathrm{Mg} / \mathrm{m}^{3}\right)\end{array}$} & $\begin{array}{c}4, \\
1.218\end{array}$ & $\begin{array}{c}2 \\
1.294\end{array}$ & $\begin{array}{c}2, \\
1.143\end{array}$ & $\begin{array}{c}8 \\
1.116\end{array}$ \\
\hline \multicolumn{2}{|c|}{ Absorption coefficient $\left(\mathrm{mm}^{-1}\right)$} & 0.567 & 0.086 & 0.067 & 0.065 \\
\hline \multicolumn{2}{|l|}{$\mathrm{F}(000)$} & 728 & 460 & 508 & 2024 \\
\hline \multicolumn{2}{|l|}{ Crystal size $(\mathrm{mm})$} & $\begin{array}{c}0.38 \times 0.07 \times \\
0.04\end{array}$ & $\begin{array}{c}0.40 \times 0.18 \times \\
0.14\end{array}$ & $\begin{array}{c}0.54 \times 0.04 \times \\
0.03\end{array}$ & $\begin{array}{c}0.20 \times 0.10 \times \\
0.08\end{array}$ \\
\hline \multicolumn{2}{|c|}{$\theta$ range for data collection $\left(^{\circ}\right)$} & 3.806 to 68.230 & 3.377 to 29.521 & 3.706 to 23.533 & 3.576 to 25.347 \\
\hline \multicolumn{2}{|l|}{ Limiting indices } & $\begin{array}{c}-11 \leq \mathrm{h} \leq 11 \\
-24 \leq \mathrm{k} \leq 27 \\
-11 \leq 1 \leq 10\end{array}$ & $\begin{array}{c}-8 \leq \mathrm{h} \leq 8 \\
-16 \leq \mathrm{k} \leq 16 \\
-19 \leq 1 \leq 21\end{array}$ & $\begin{array}{c}-10 \leq \mathrm{h} \leq 10 \\
-13 \leq \mathrm{k} \leq 14 \\
-11 \leq 1 \leq 14\end{array}$ & $\begin{array}{c}-30 \leq \mathrm{h} \leq 31 \\
-11 \leq \mathrm{k} \leq 10 \\
-30 \leq 1 \leq 30\end{array}$ \\
\hline \multicolumn{2}{|c|}{$\begin{array}{l}\text { Reflections collected / unique } \\
\text { R(int) }\end{array}$} & $\begin{array}{c}12735 / 5512 \\
0.0226\end{array}$ & $\begin{array}{c}22164 / 5648 \\
0.0798\end{array}$ & $\begin{array}{c}25880 / 4054 \\
0.161\end{array}$ & $\begin{array}{c}22649 / 5136 \\
0.046\end{array}$ \\
\hline \multicolumn{2}{|c|}{ Completeness to $\theta_{\text {full }}(\%)$} & 97.0 & 99.5 & $\begin{array}{c}98.9 \\
\left(\text { at } \theta_{\max } 23.5^{\circ}\right)\end{array}$ & 99.6 \\
\hline \multicolumn{2}{|l|}{ Absorption correction } & \multicolumn{4}{|c|}{ Semi-empirical from equivalents } \\
\hline \multicolumn{2}{|c|}{ Max. and min. transmission } & 1.000 and 0.841 & 1.000 and 0.365 & 1.000 and 0.349 & $\begin{array}{l}1.000 \text { and } \\
0.225\end{array}$ \\
\hline \multicolumn{2}{|l|}{ Refinement method } & \multicolumn{4}{|c|}{ Full-matrix least-squares on $F^{2}$} \\
\hline \multicolumn{2}{|c|}{ Data / restraints / parameters } & $5504 / 5 / 499$ & $5638 / 0 / 374$ & $4031 / 1 / 339$ & $5135 / 55 / 337$ \\
\hline \multicolumn{2}{|l|}{ Goodness-of-fit on $F^{2}$} & 1.149 & 1.031 & 1.126 & 1.032 \\
\hline \multicolumn{2}{|c|}{ Final $\mathrm{R}$ indices $[I>2 \sigma(I)]$} & $\begin{array}{l}0.0411 \\
0.0857\end{array}$ & $\begin{array}{l}0.0657 \\
0.1547\end{array}$ & $\begin{array}{l}0.095 \\
0.220\end{array}$ & $\begin{array}{l}0.069 \\
0.175\end{array}$ \\
\hline \multicolumn{2}{|l|}{$\mathrm{R}$ indices (all data) } & $\begin{array}{r}0.1005 \\
0.1371\end{array}$ & $\begin{array}{l}0.1124 \\
0.1725\end{array}$ & $\begin{array}{l}0.118 \\
0.233\end{array}$ & $\begin{array}{l}0.091 \\
0.189\end{array}$ \\
\hline \multicolumn{2}{|c|}{ Absolute structure parameter } & $0.4(3)$ & - & - & - \\
\hline \multicolumn{2}{|l|}{ Extinction coefficient } & - & - & $0.161(17)$ & - \\
\hline \multicolumn{2}{|c|}{$\begin{array}{l}\text { Largest diff. peak and hole (e. } \\
\AA^{3} \text { ) }\end{array}$} & $\begin{array}{l}0.228 \text { and - } \\
0.229\end{array}$ & $\begin{array}{l}0.233 \text { and - } \\
0.224\end{array}$ & $\begin{array}{l}0.309 \text { and - } \\
0.292\end{array}$ & $\begin{array}{l}0.248 \text { and - } \\
0.299\end{array}$ \\
\hline
\end{tabular}




\section{References}

1 Rigaku - Higashi T. FS Process, Rigaku Corporation,Tokyo, Japan, 1998.

2 Rigaku, CrystalClear-SM Expert 2.0 r4 Rigaku Corporation, Tokyo, Japan, 2009.

3 Rigaku OD, CrysAlis PRO. Rigaku Oxford Diffraction, Yarnton, Oxfordshire, England, 2015.

4 Sheldrick, G. M. SHELXT - Integrated space-group and crystal-structure determination. Acta Crystallogr. 2015, A71, 3-8.

5 Sheldrick, G. M. Crystal structure refinement with SHELXL. Acta Crystallogr. 2015, C71, 3-8.

6 Spek, A. L. Structure validation in chemical crystallography. Acta Cryst. 2009, D65, 148-155.

7 Bernstein, J.; Davis, R. E.; Shimoni, L; Chang, N. L. Patterns in Hydrogen Bonding: Functionality and Graph Set Analysis in Crystals. Angew. Chem. Int. Ed. 1995, 34, 1555-1573. 


\section{Procedure for large scale synthesis of $2 a$}

A mixture of phenylacetonitrile 1a (1.17 g, $10 \mathrm{mmol})$, sulfur (320 mg, $10 \mathrm{mmol}), \mathrm{DABCO}(224 \mathrm{mg}, 2$ $\mathrm{mmol})$ and DMSO $(1 \mathrm{~mL})$ and a magnetic stir bar was added to a $17-\mathrm{mL}$ test tube (Figure S3a). The tube was closed with a rubber septum. Air inside the tube was replaced by an argon atmosphere (three times rotavapor pump/Ar) and the tube was heated at $80{ }^{\circ} \mathrm{C}$ for $16 \mathrm{~h}$ (Figure $\mathbf{S 3 b}$ ). The reaction was cooled to rt, methanol $(6 \mathrm{~mL})$ was added (Figure S3c). The resulting mixture was shaken (vortex mixer), filtered. The yellow residue was washed with methanol $(20 \mathrm{~mL})$ (Figure S3d). The solid was dried in vacuo $(0.01 \mathrm{mmHg})$ at $100{ }^{\circ} \mathrm{C}$. The yield of $2 \mathbf{a}$ was $1.08 \mathrm{~g}(93 \%)$.
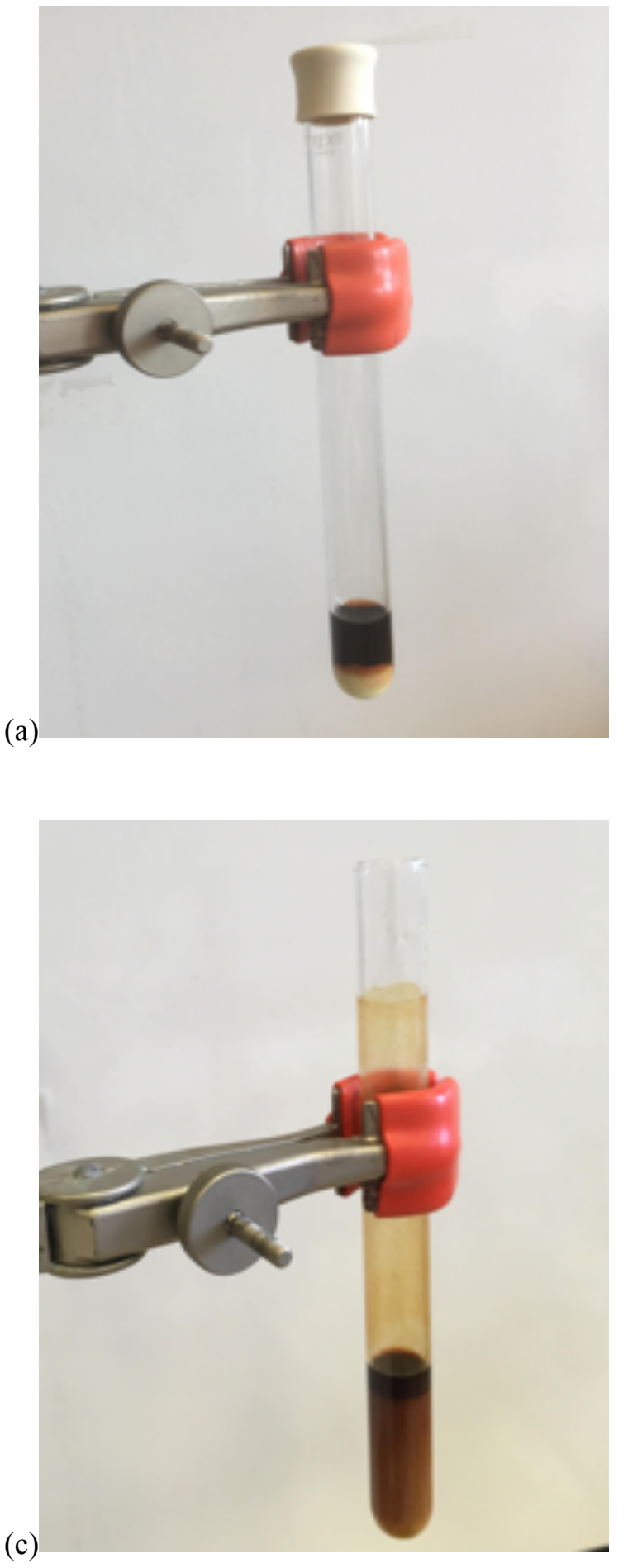

(b)

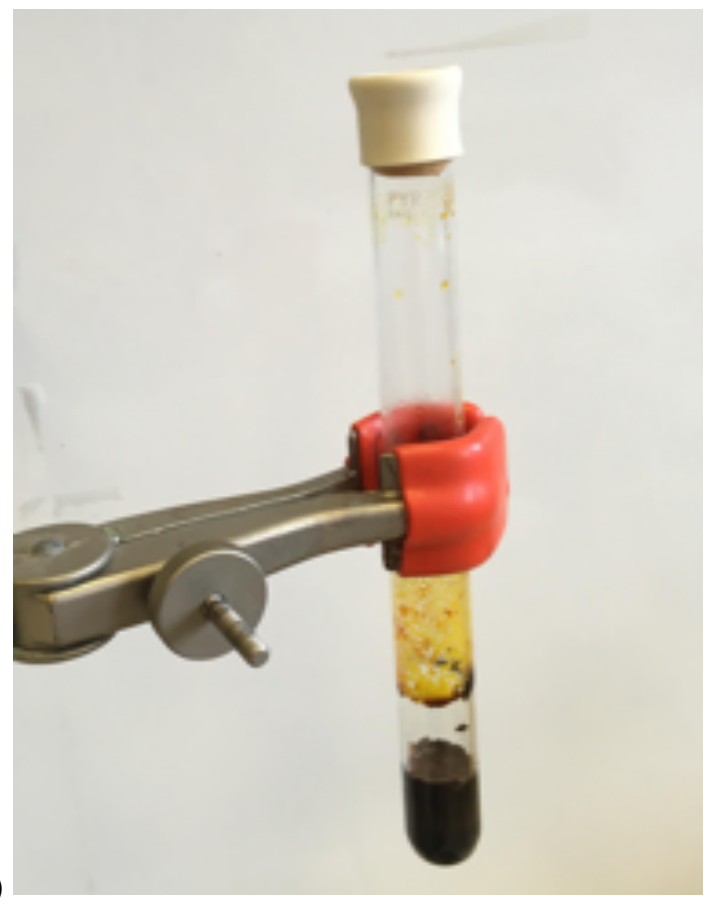

(d)

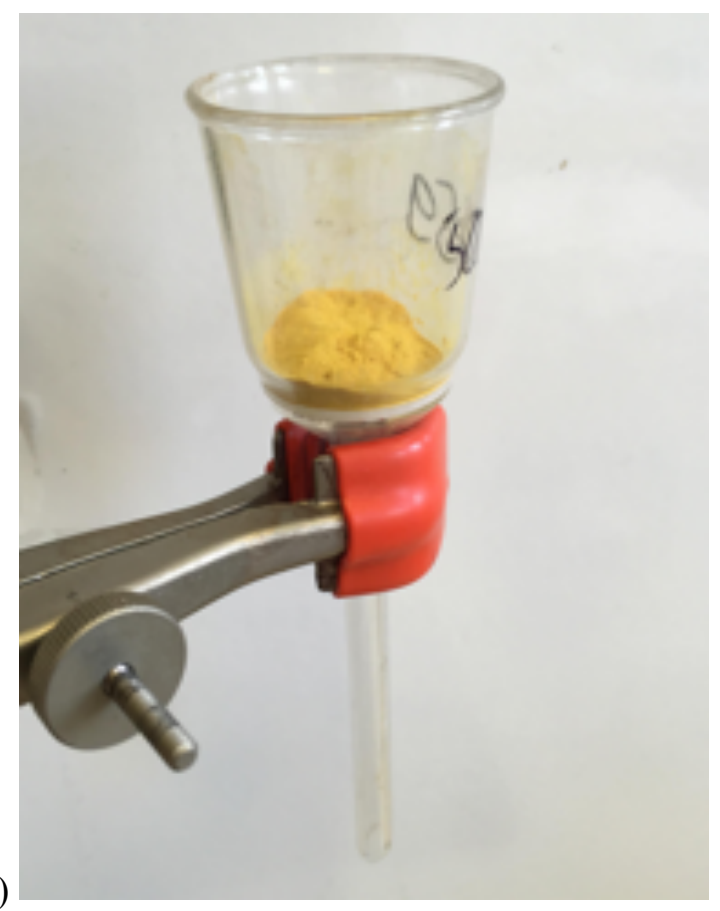

Figure S3. (a) Reaction mixture before heating; (b) Reaction mixture after heating at $80{ }^{\circ} \mathrm{C}$ for $16 \mathrm{~h}$; (c) After adding methanol (6 mL); (d) After filtration and washing with methanol. 


\section{Copies of ${ }^{1} \mathrm{H}$ and ${ }^{13} \mathrm{C}$ NMR spectra}

(E)-2-(5-Amino-3,4-diphenyl-2H-pyrrol-2-ylidene)-2-phenylacetonitrile (2a)

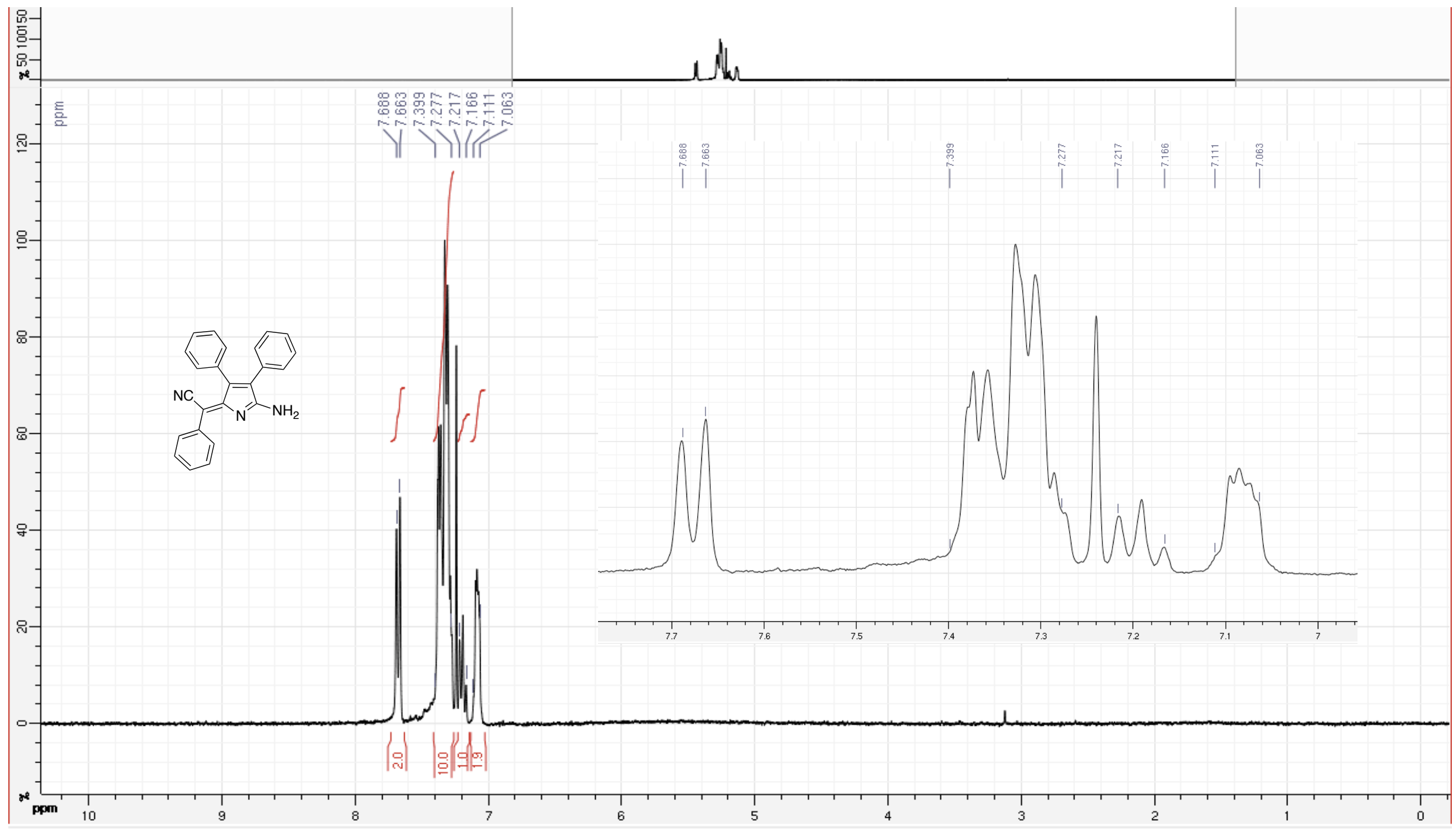




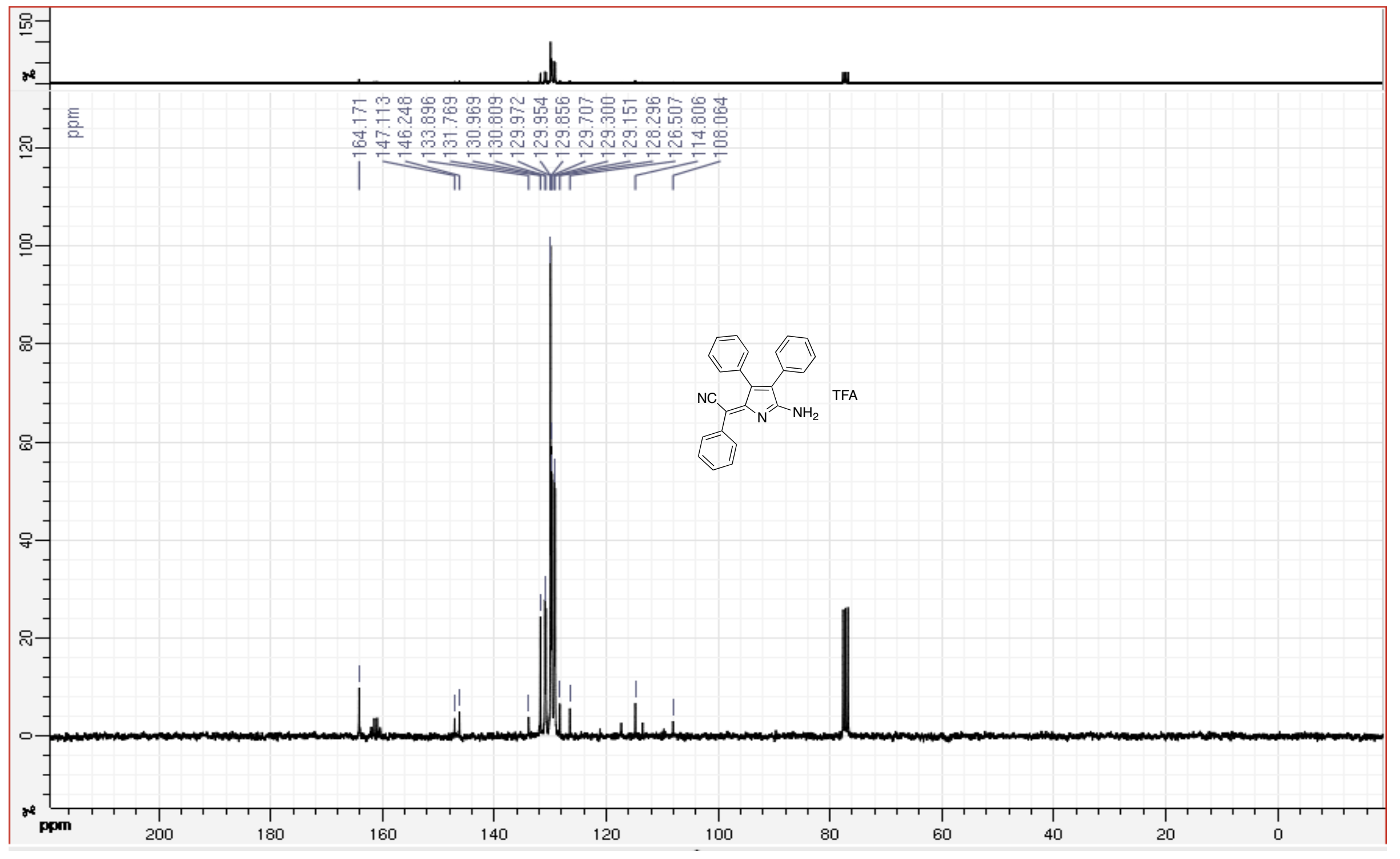


(E)-2-(5-Amino-3,4-di-p-tolyl-2H-pyrrol-2-ylidene)-2-(p-tolyl)acetonitrile (2b)

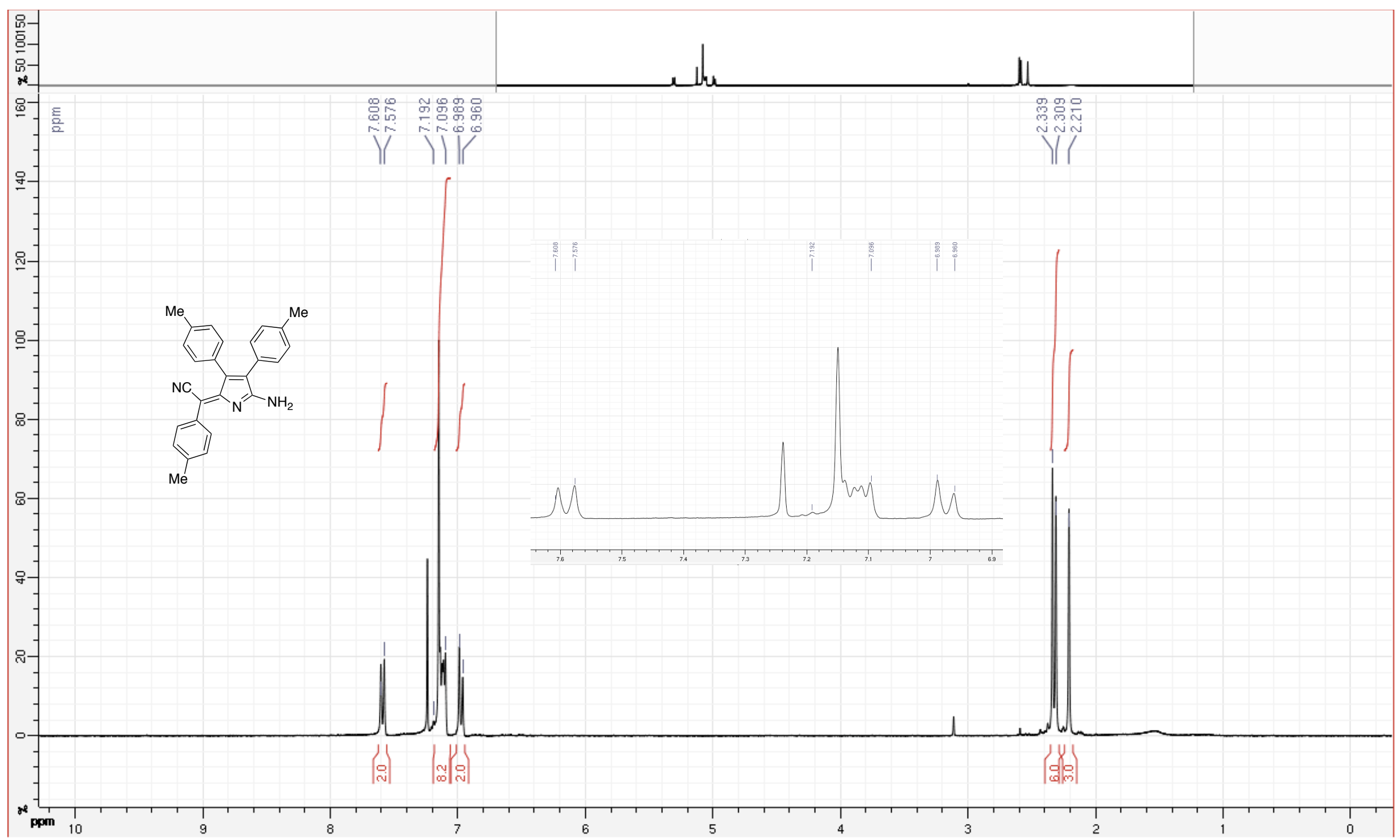




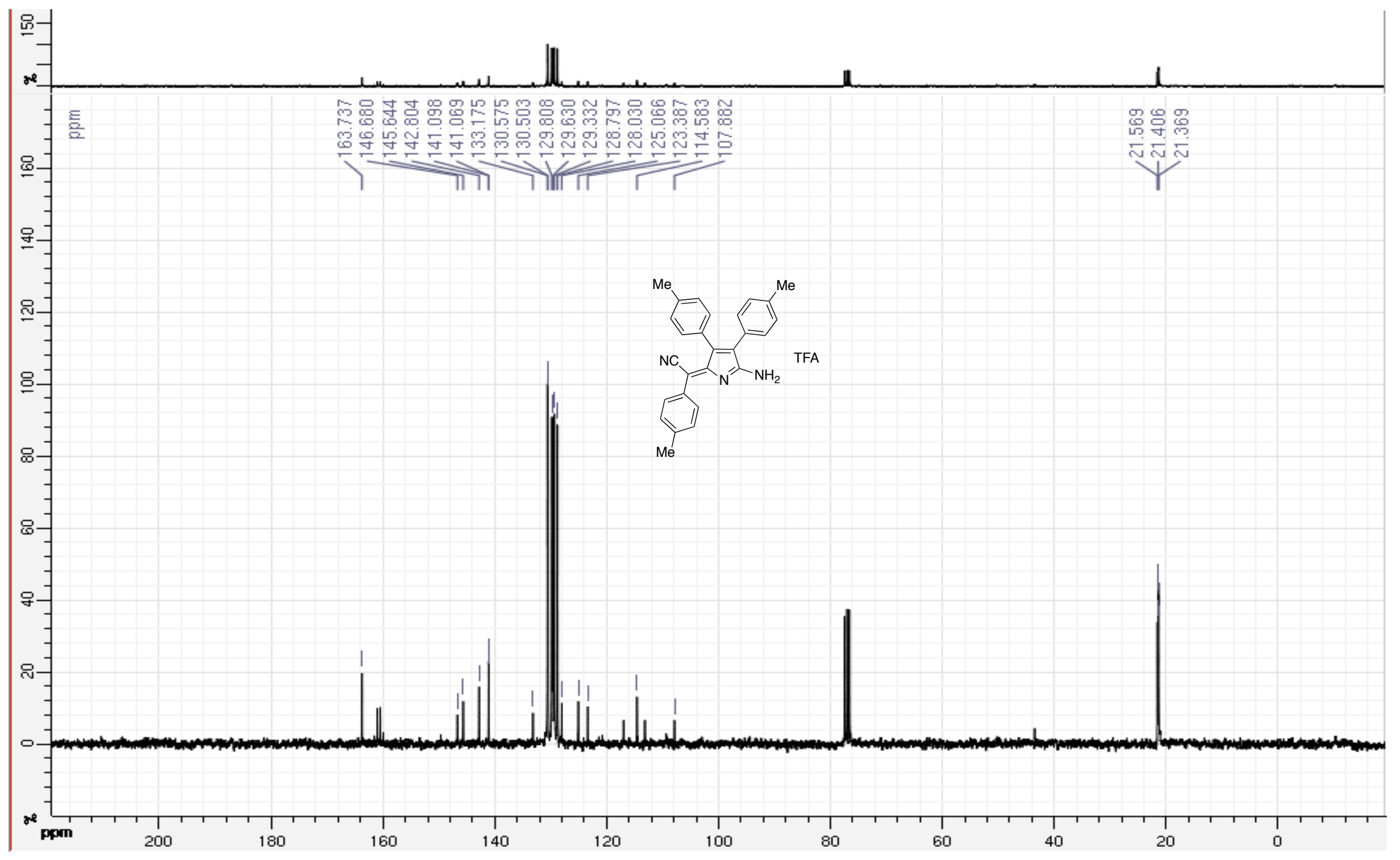


(E)-2-(5-Amino-3,4-bis(4-isopropylphenyl)-2H-pyrrol-2-ylidene)-2-(4-isopropylphenyl)acetonitrile (2c)

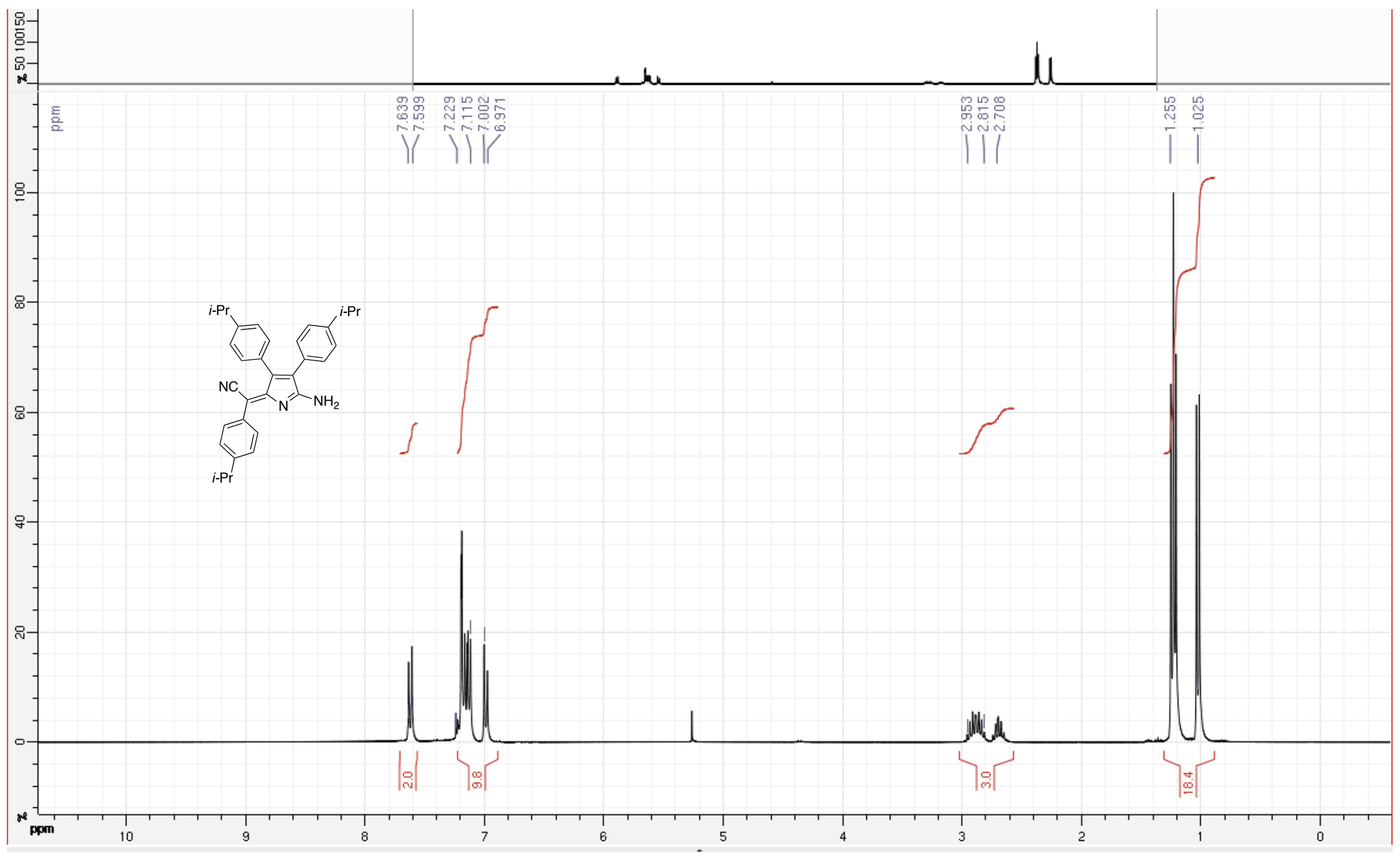




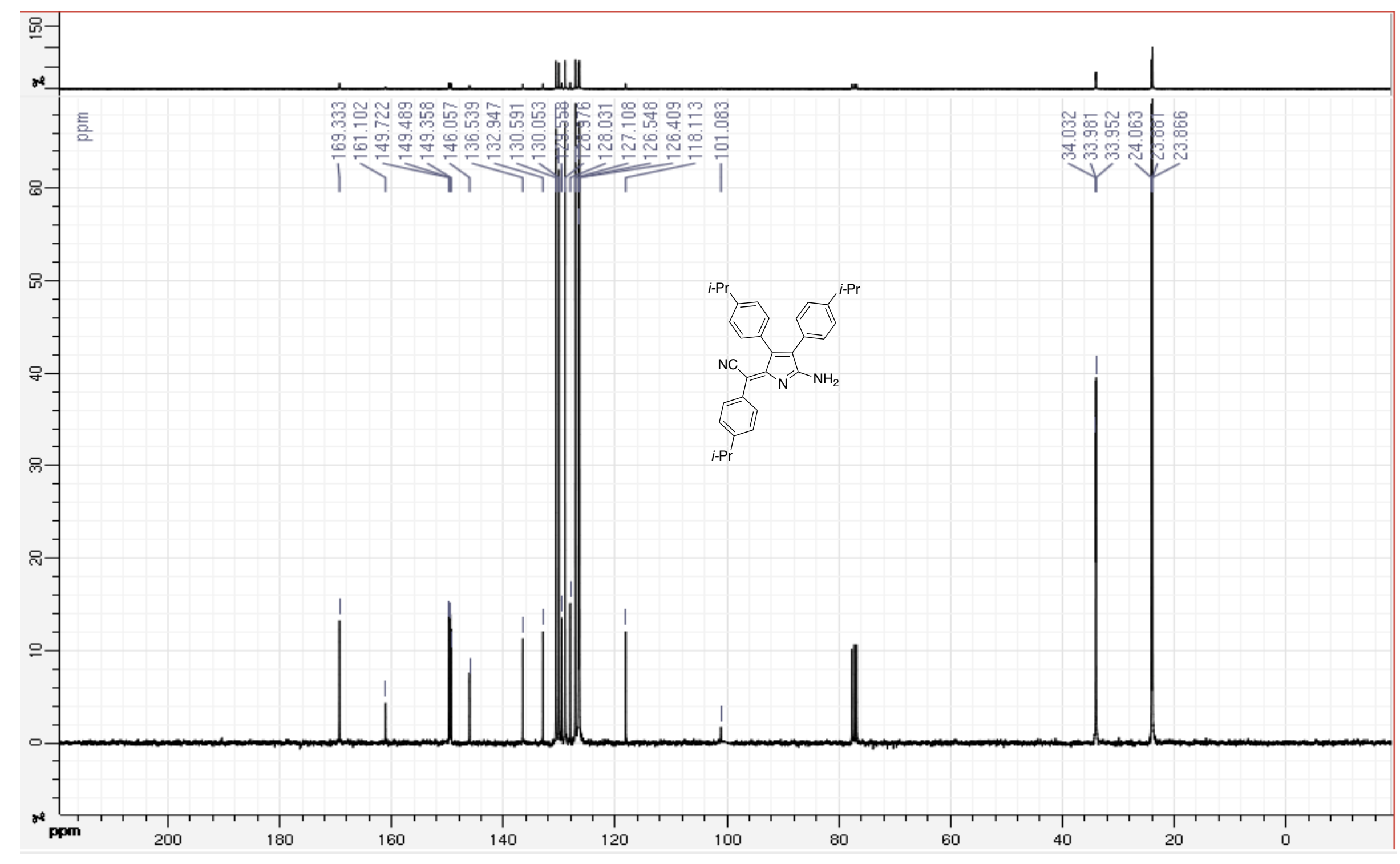


(E)-2-(5-Amino-3,4-di-m-tolyl-2H-pyrrol-2-ylidene)-2-(m-tolyl)acetonitrile (2d)

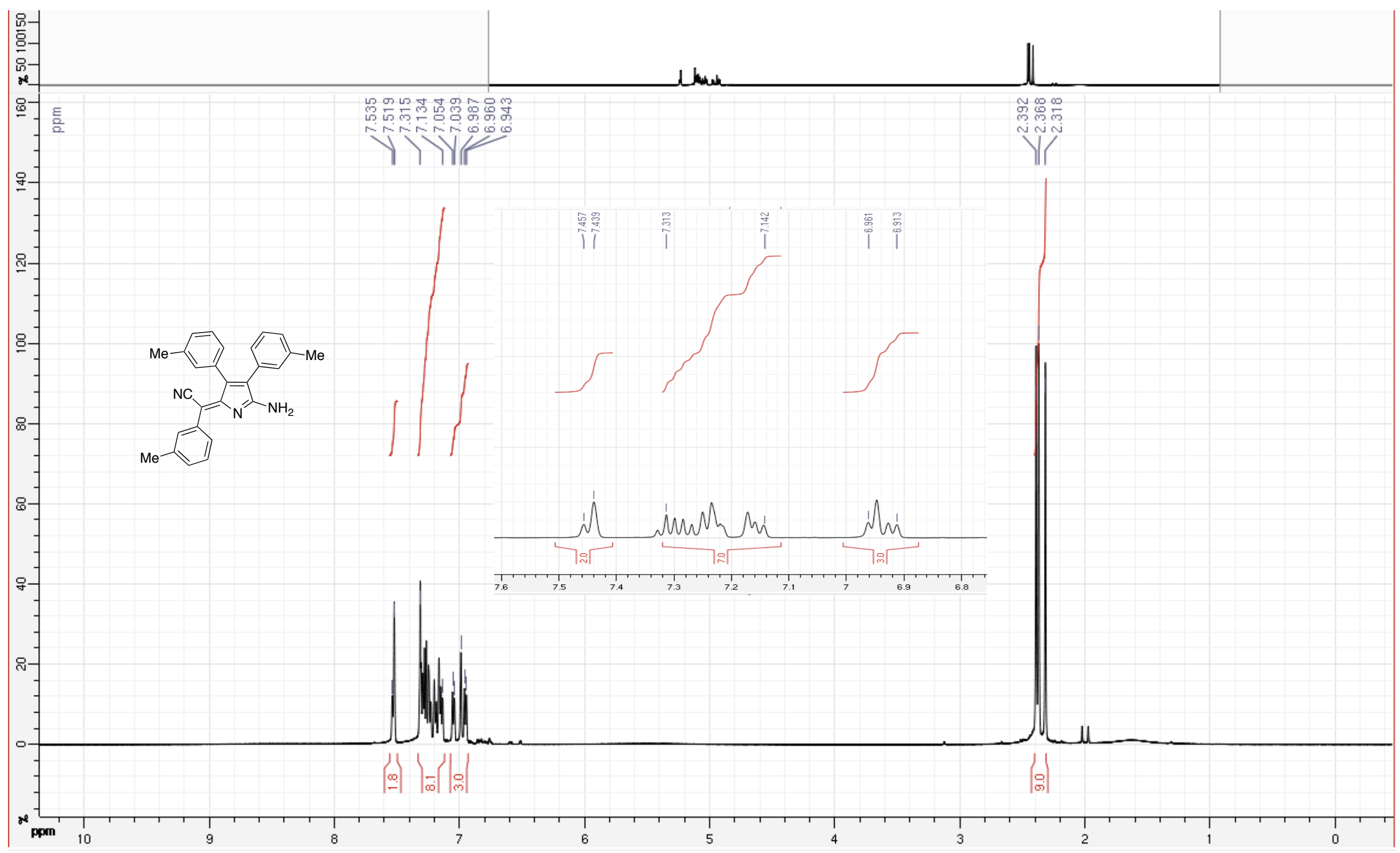




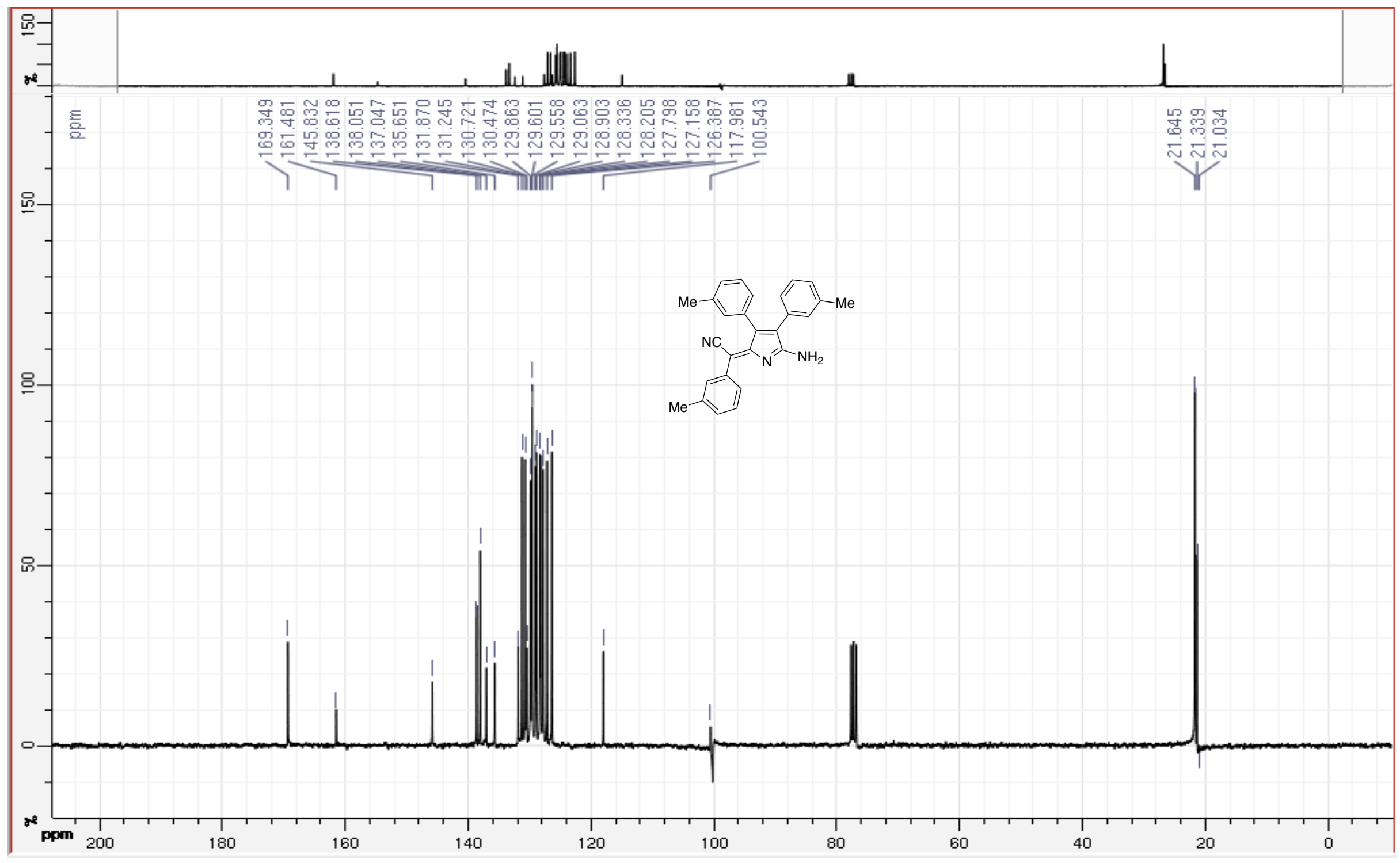


(E)-2-(5-Amino-3,4-bis(4-methoxyphenyl)-2H-pyrrol-2-ylidene)-2-(4-methoxyphenyl)acetonitrile (2e)

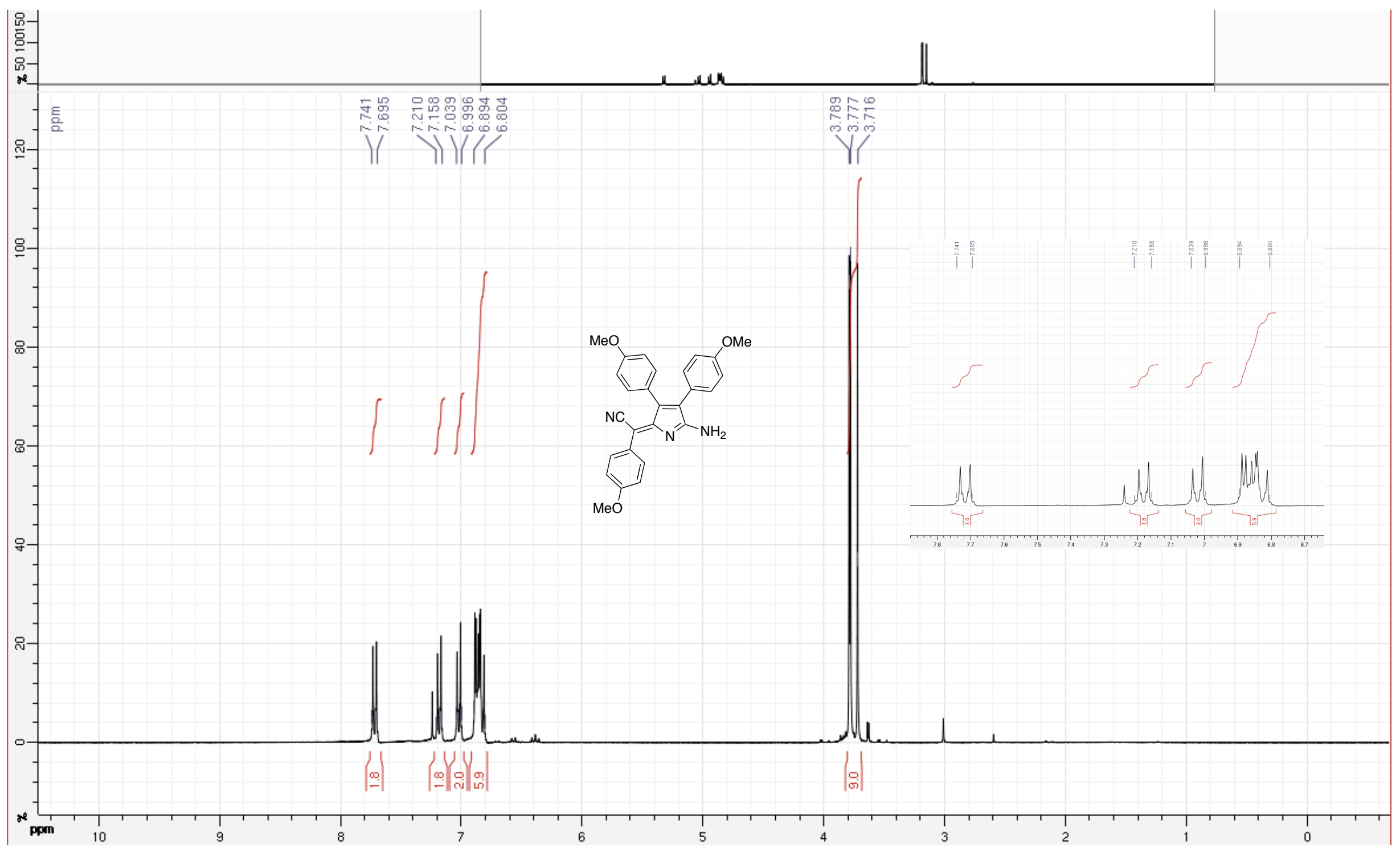




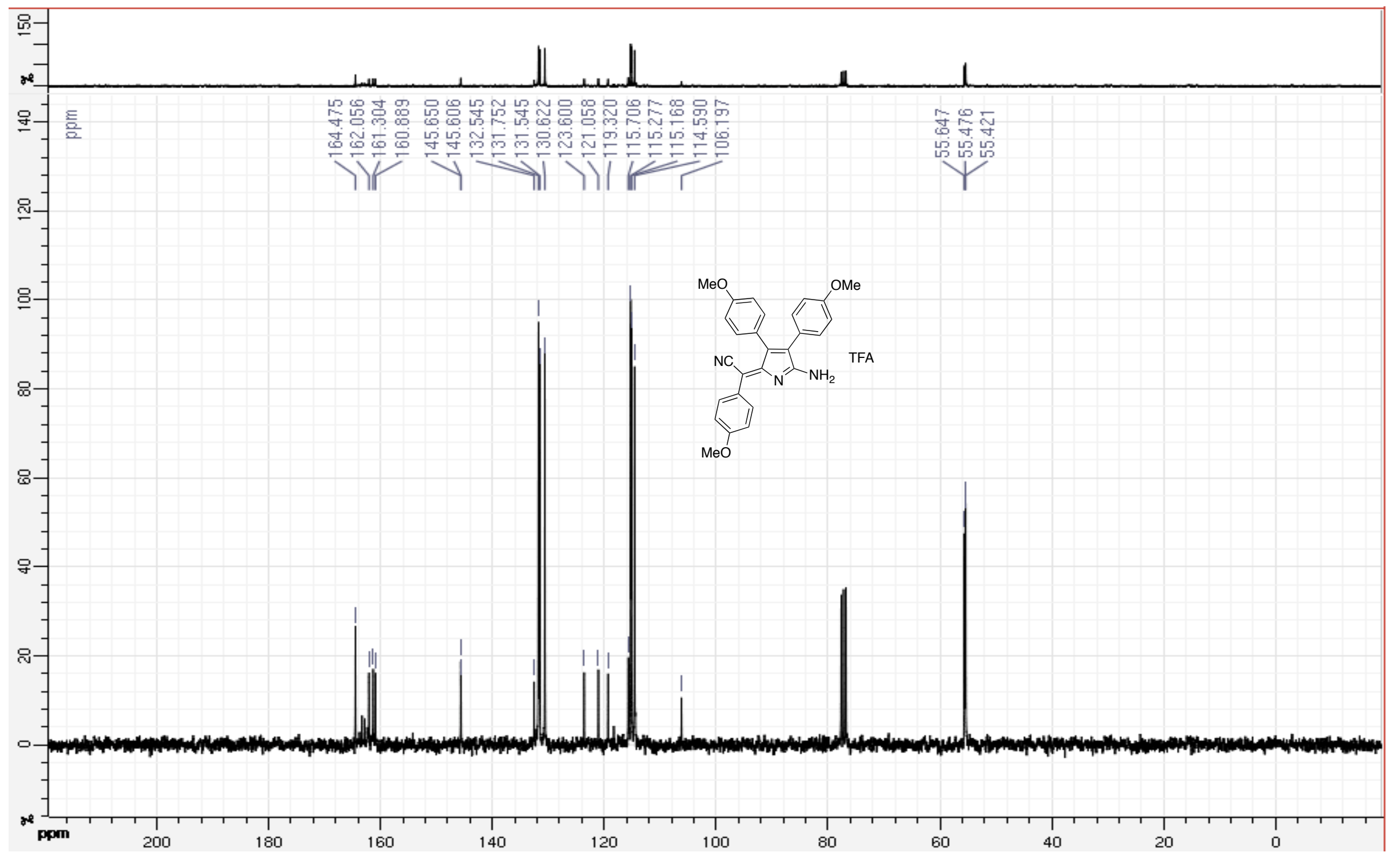


(E)-2-(5-Amino-3,4-bis(3-methoxyphenyl)-2H-pyrrol-2-ylidene)-2-(3-methoxyphenyl)acetonitrile (2f)

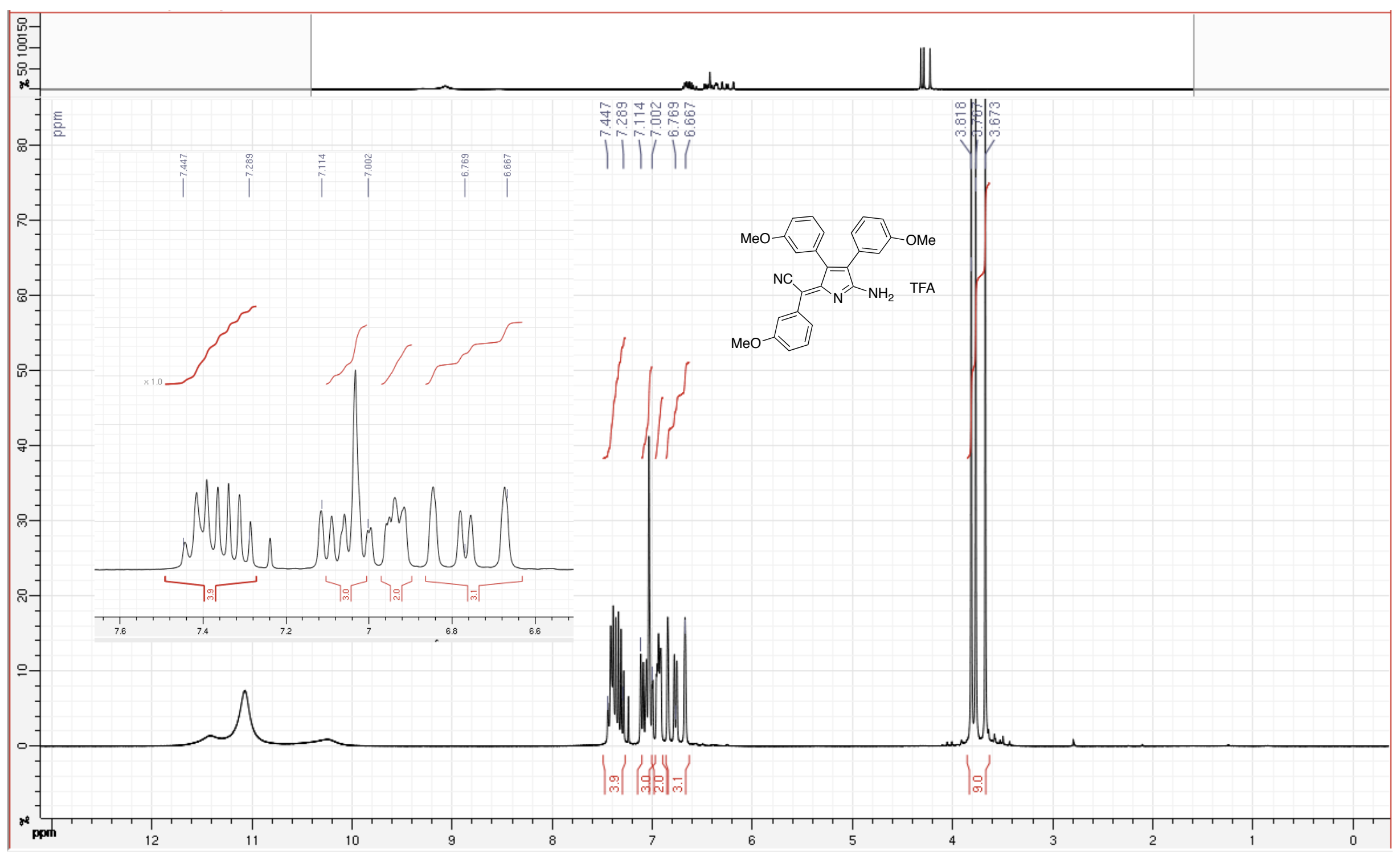




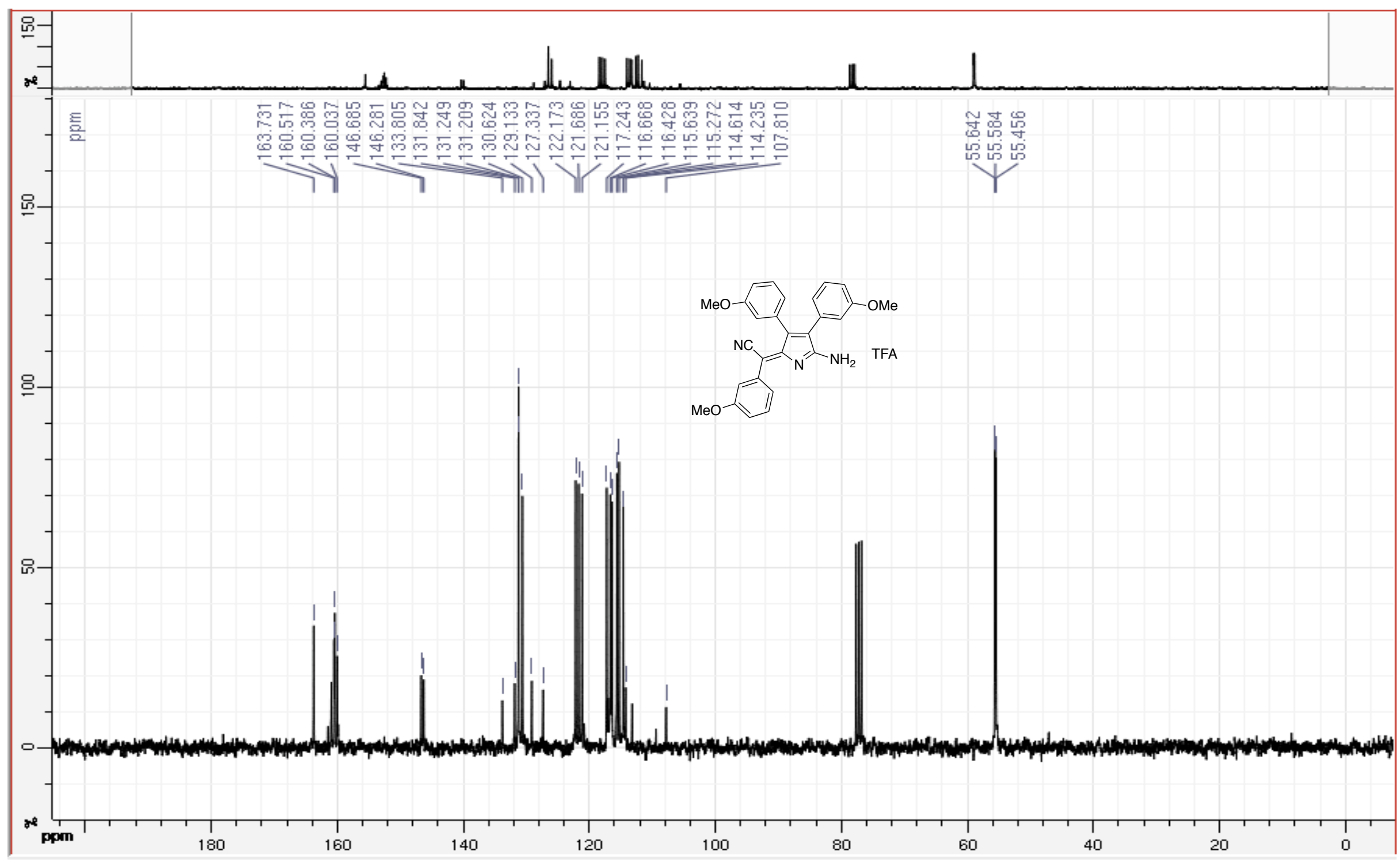


(E)-2-(5-Amino-3,4-bis(3,4-dimethoxyphenyl)-2H-pyrrol-2-ylidene)-2-(3,4-dimethoxyphenyl)acetonitrile (2g)

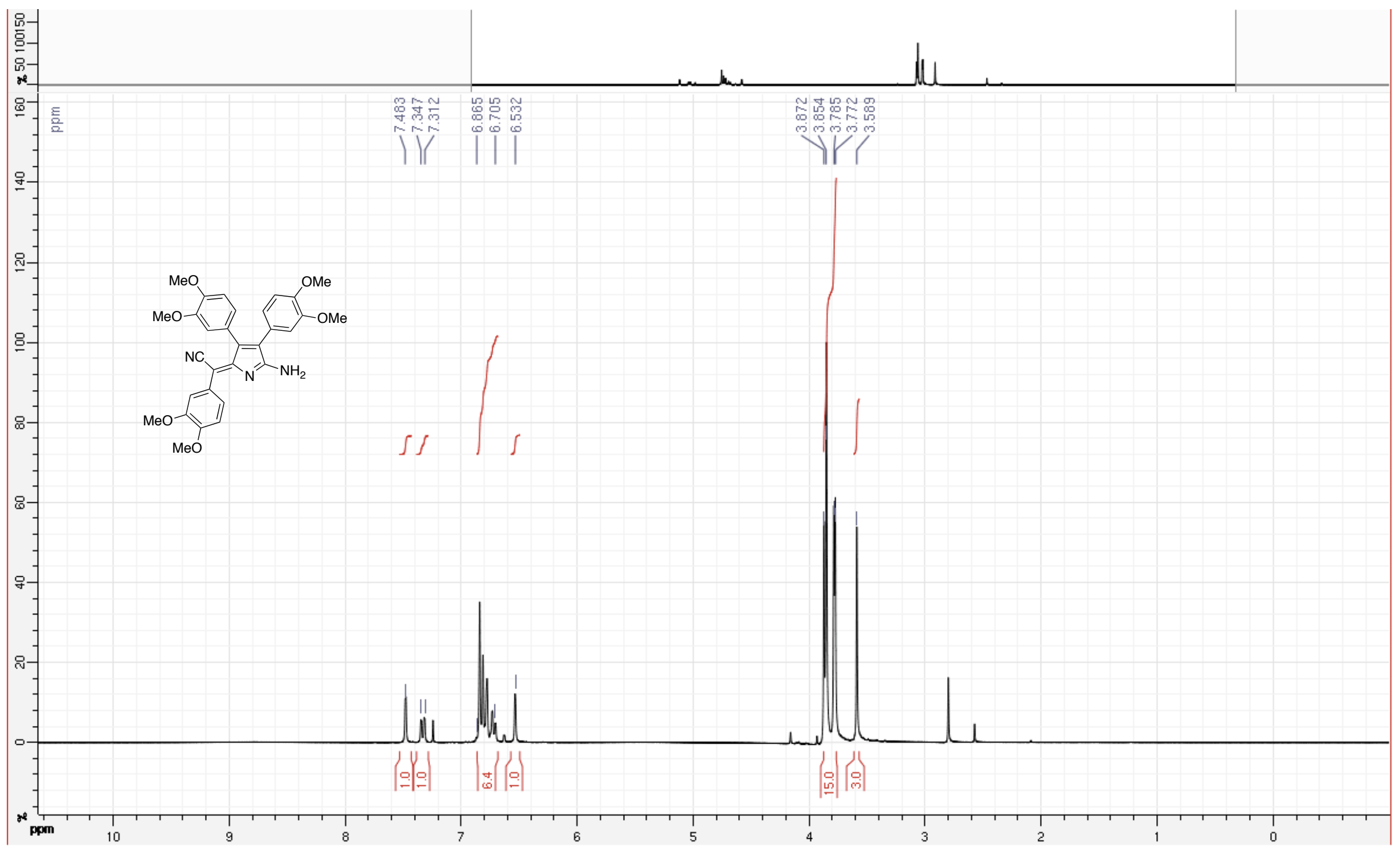




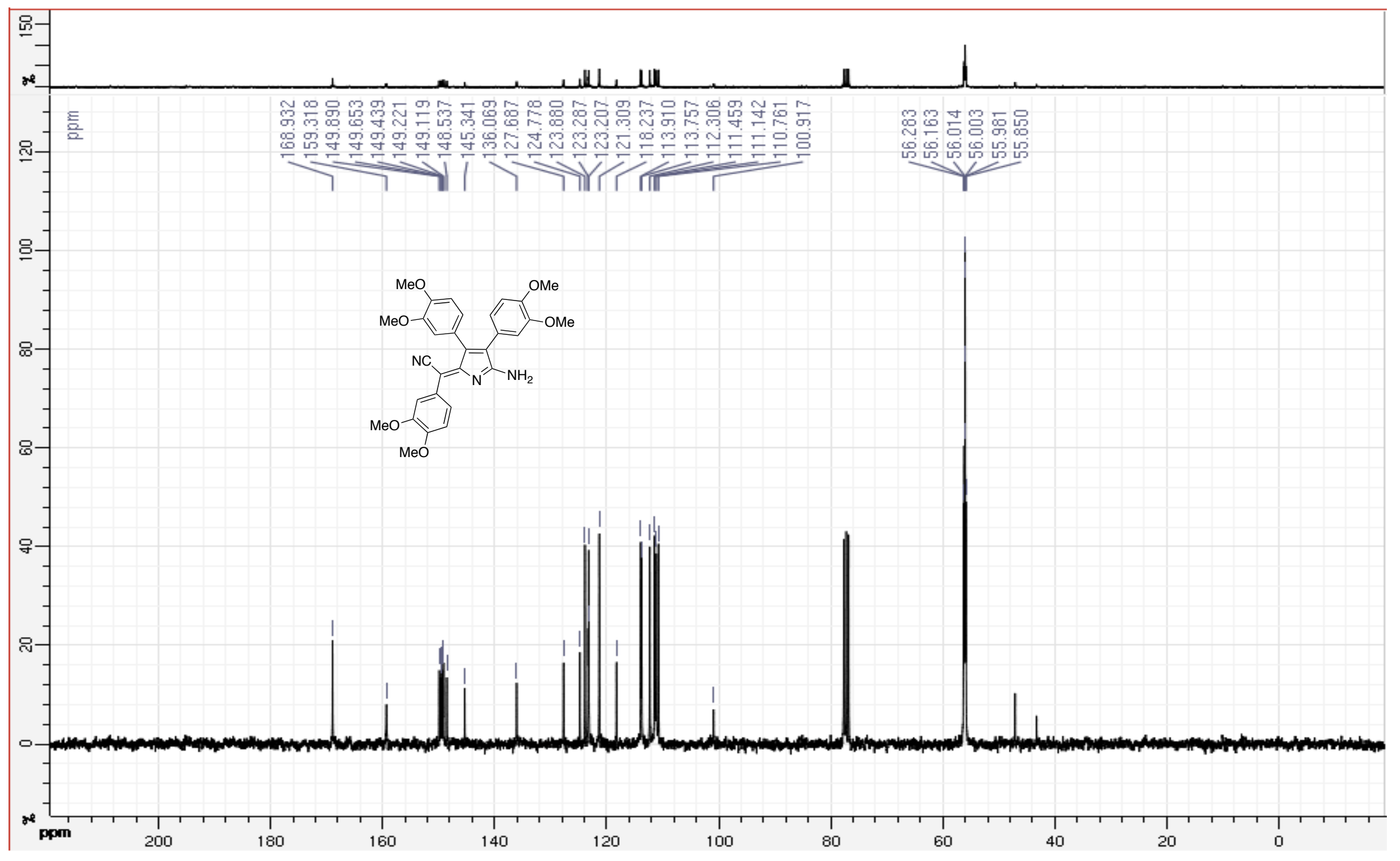


(E)-N,N'-((2-((4-Acetamidophenyl)(cyano)methylene)-5-amino-2H-pyrrole-3,4-diyl)bis(4,1-phenylene))diacetamide (2h)

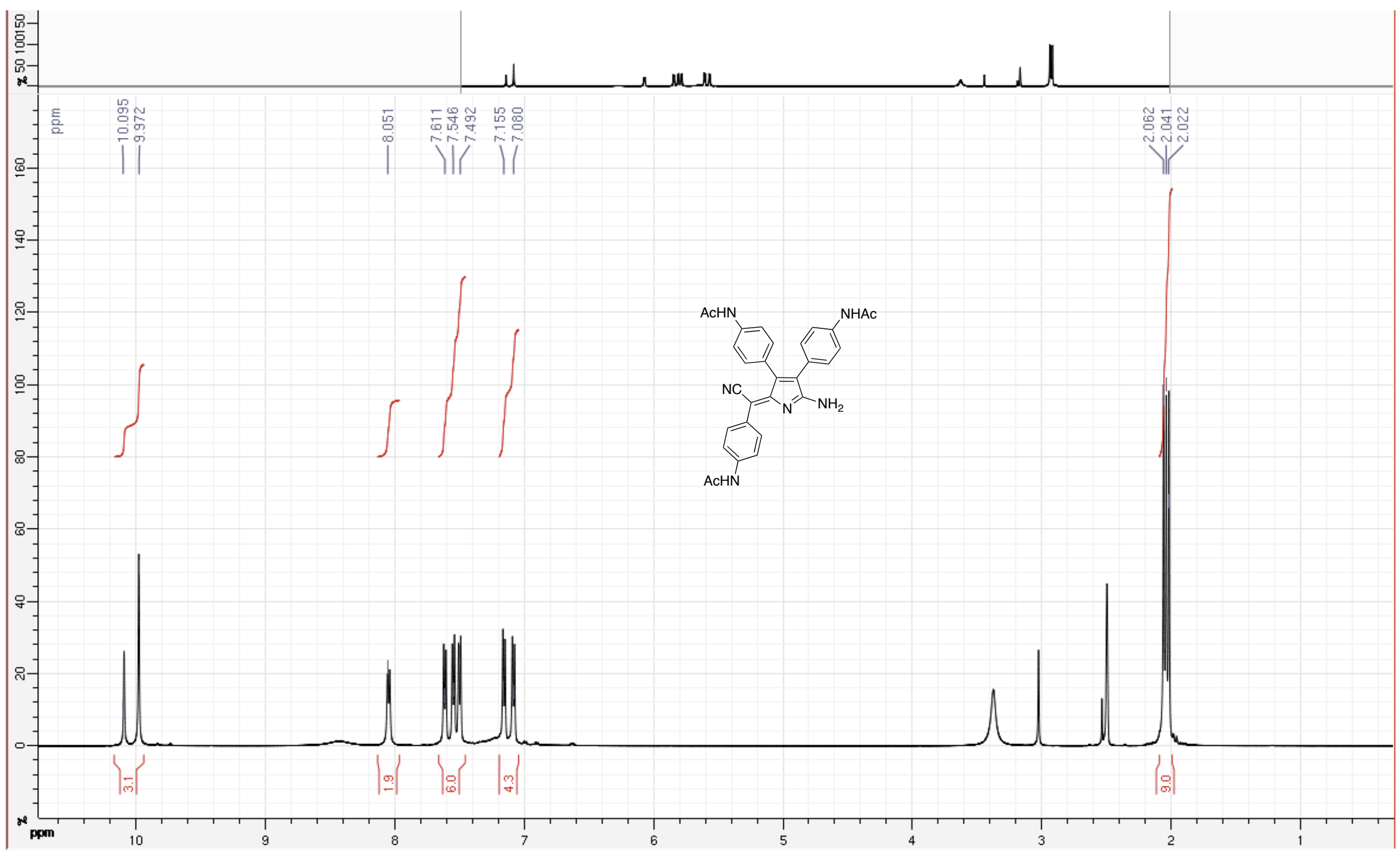




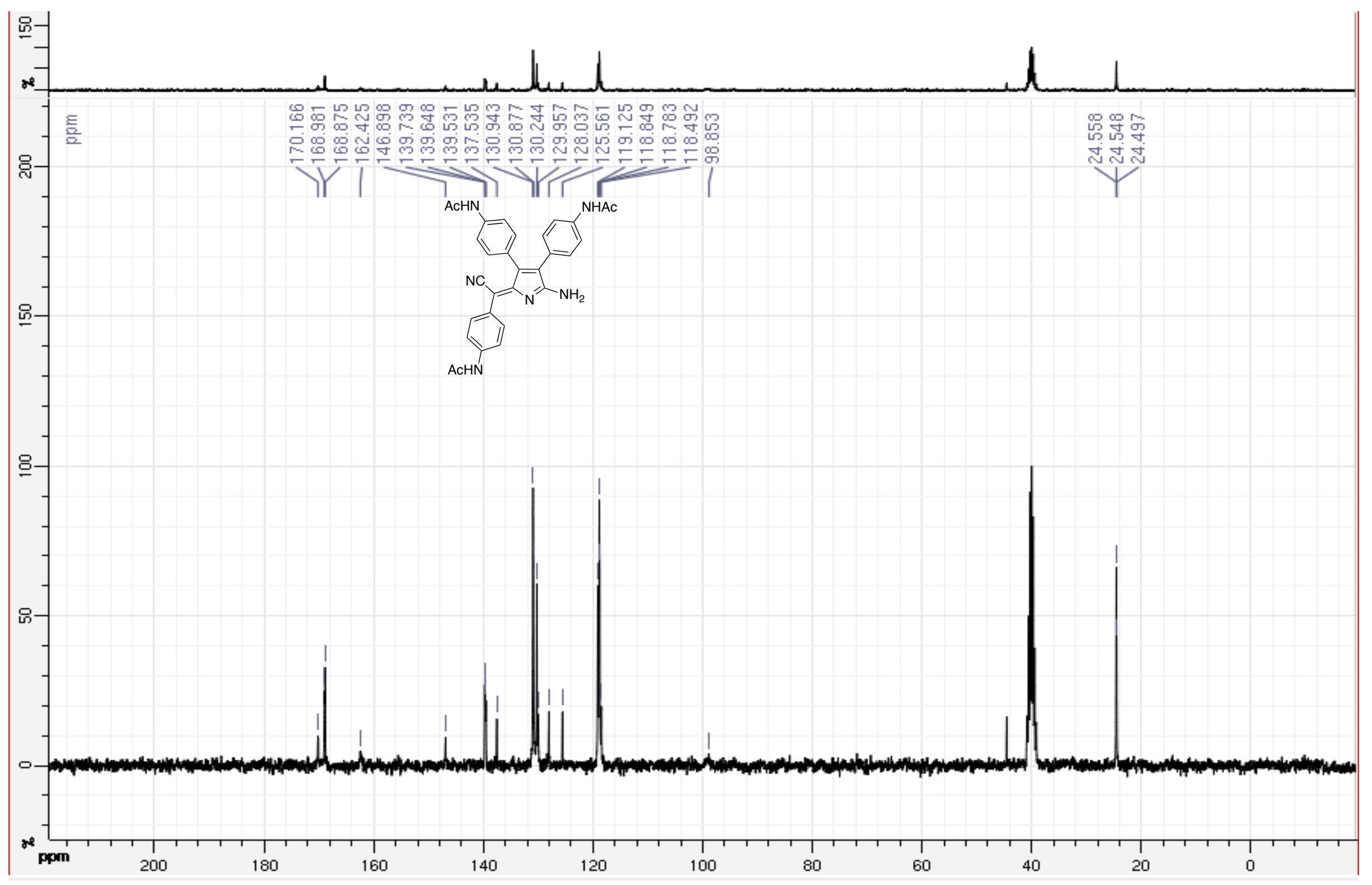


(E)-2-([1,1'-Biphenyl]-4-yl)-2-(3,4-di([1,1'-biphenyl]-4-yl)-5-amino-2H-pyrrol-2-ylidene)acetonitrile (2i)

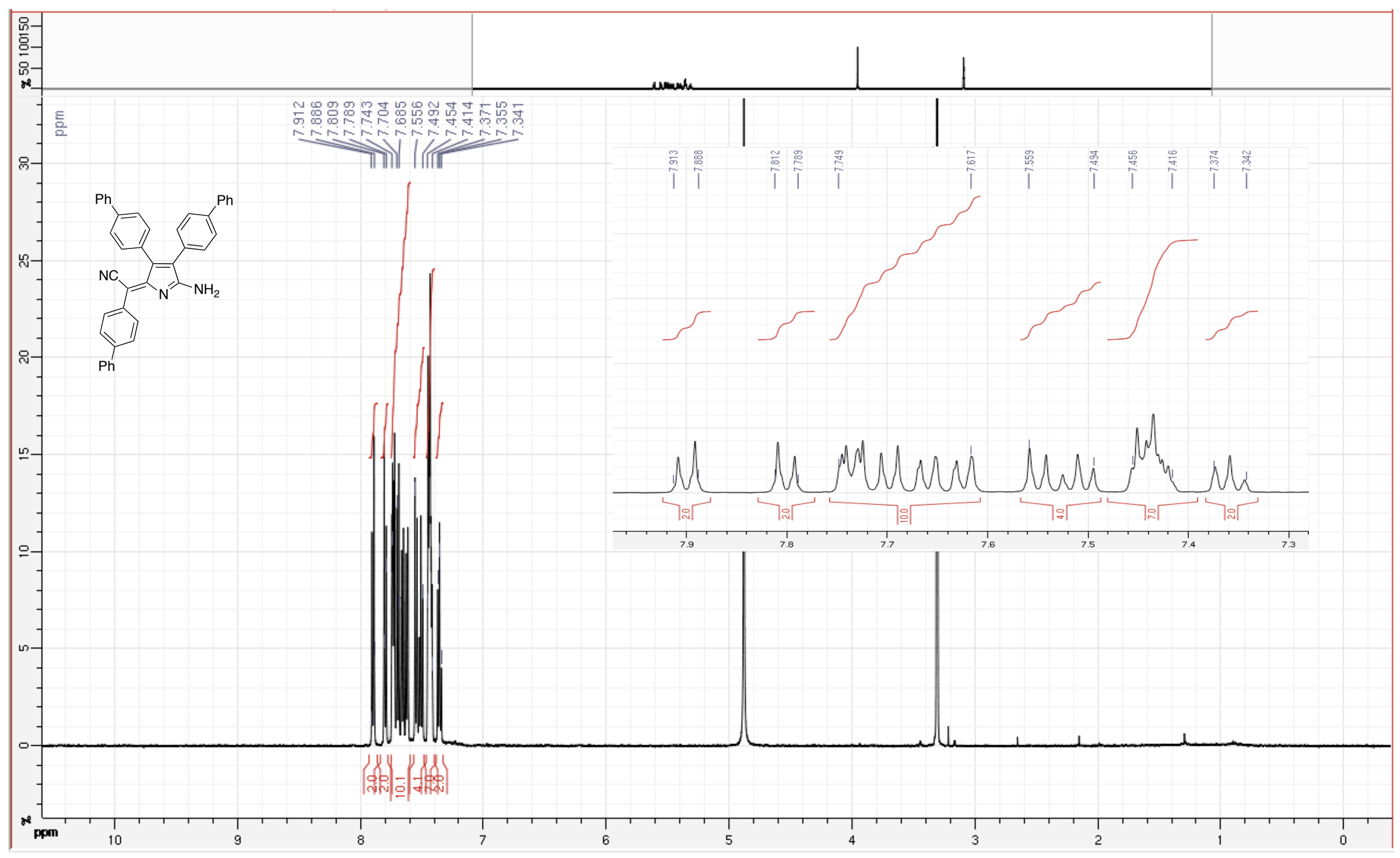




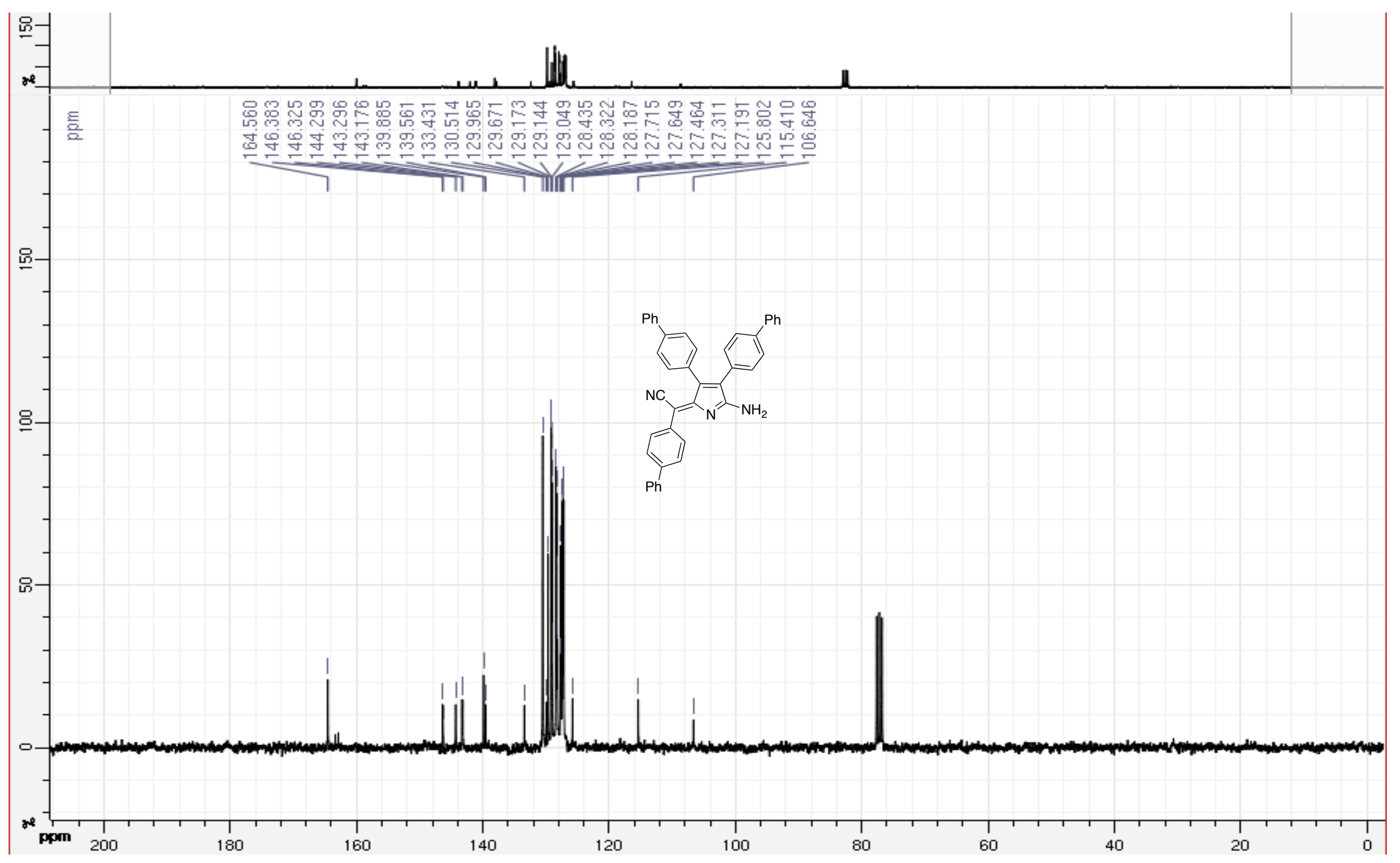


(E)-2-(5-Amino-3,4-bis(4-(trifluoromethyl)phenyl)-2H-pyrrol-2-ylidene)-2-(4-(trifluoromethyl)phenyl)acetonitrile (2j)

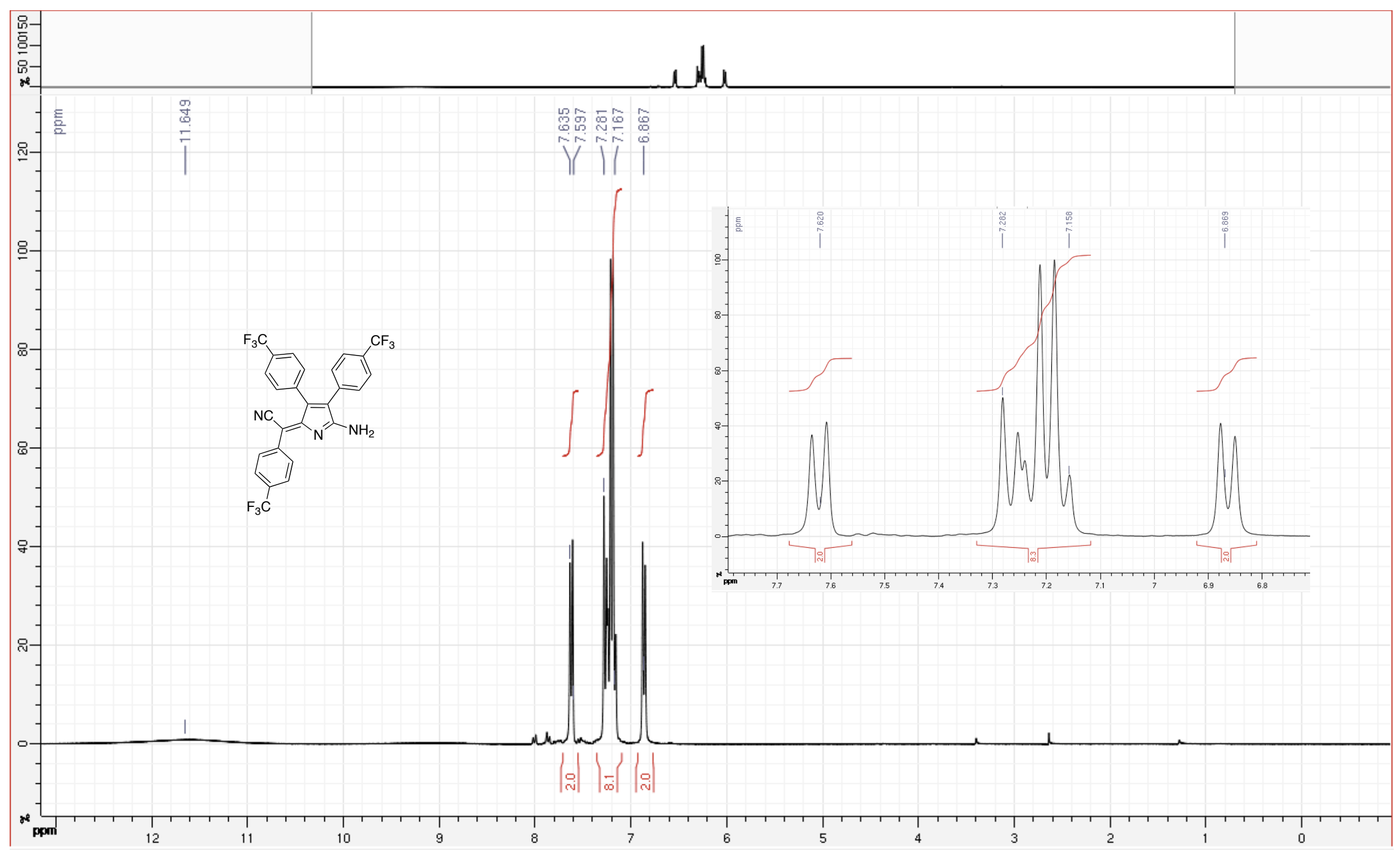




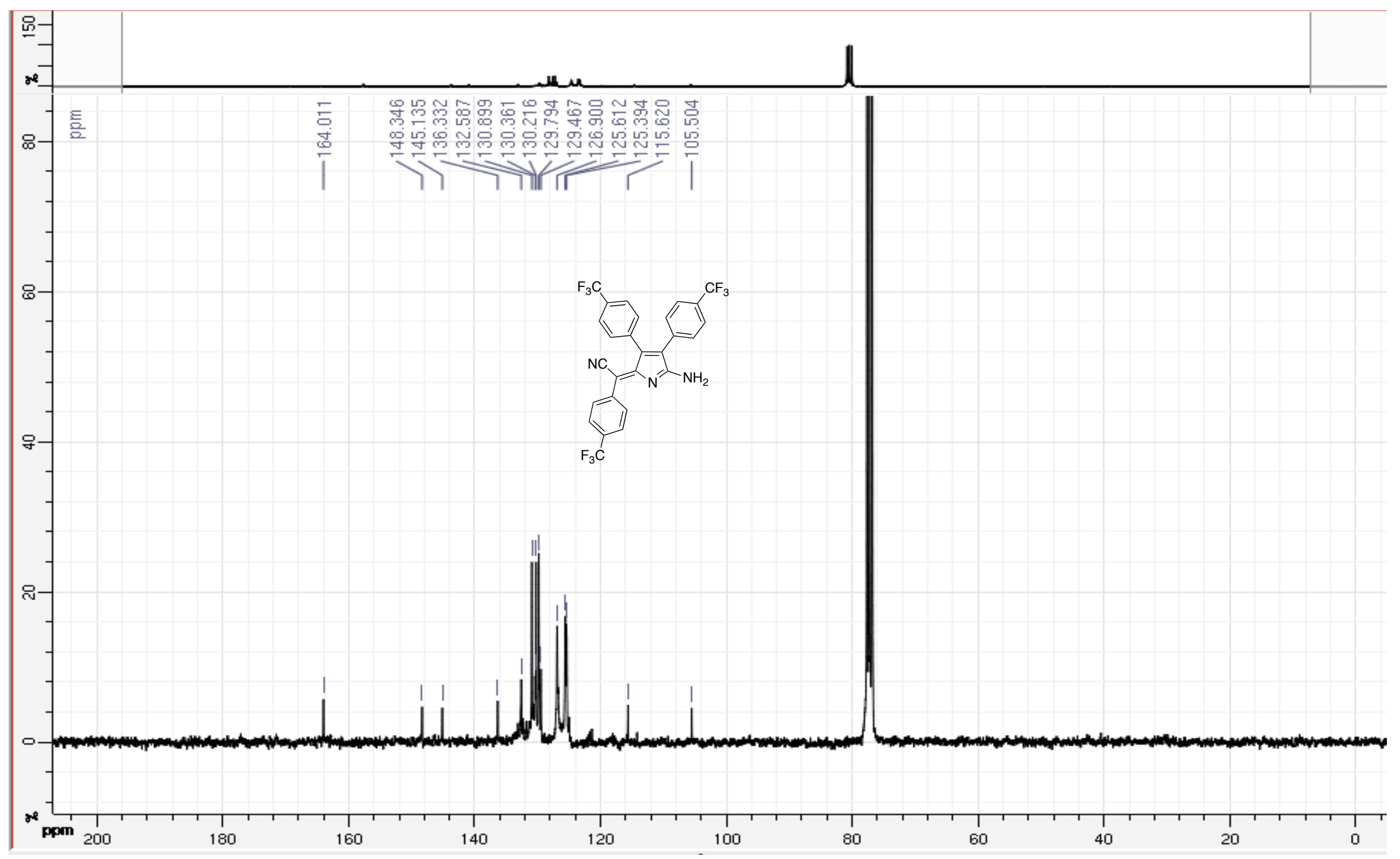


(E)-4,4'-(5-Amino-2-(cyano(4-cyanophenyl)methylene)-2H-pyrrole-3,4-diyl)dibenzonitrile (2k)

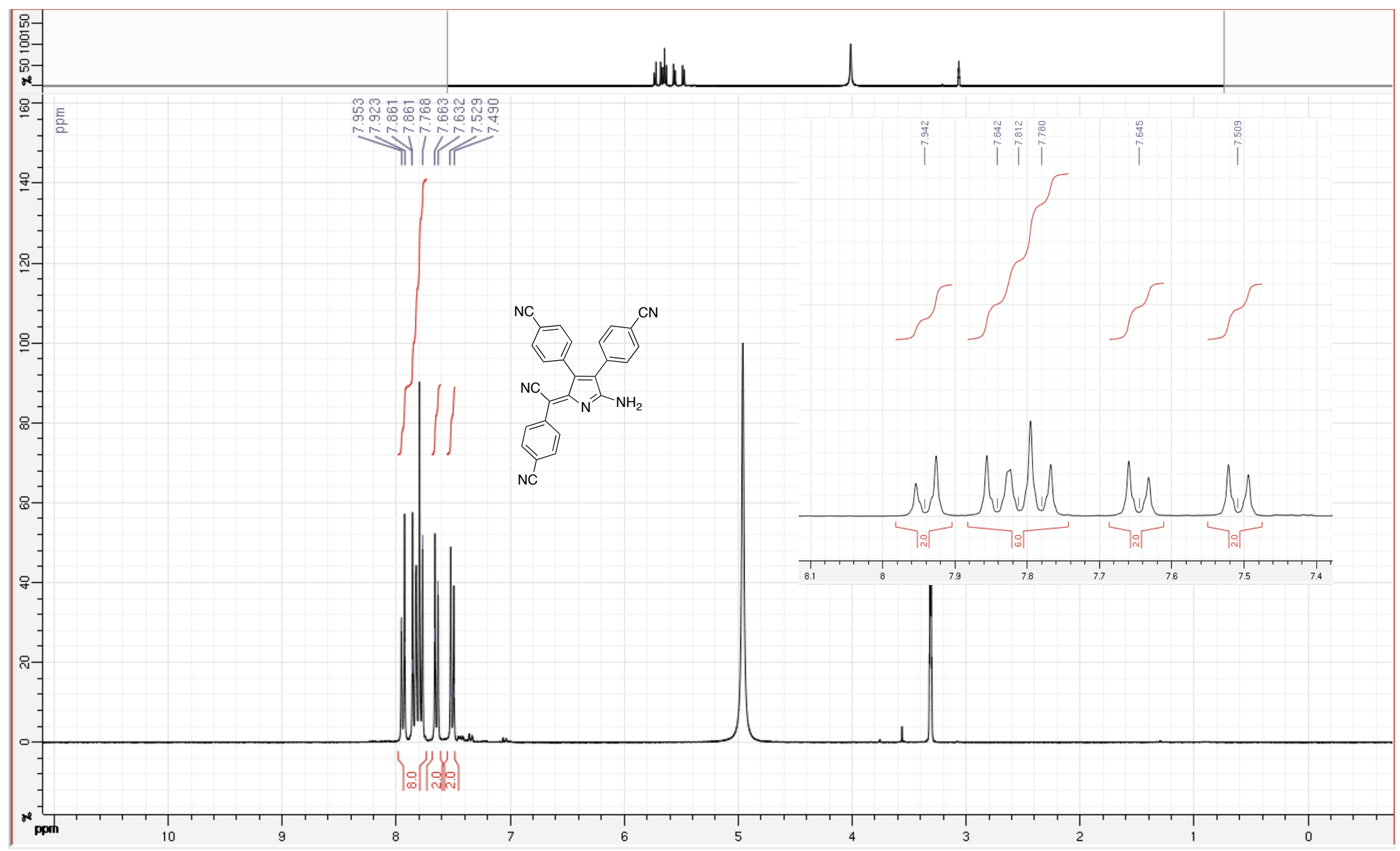




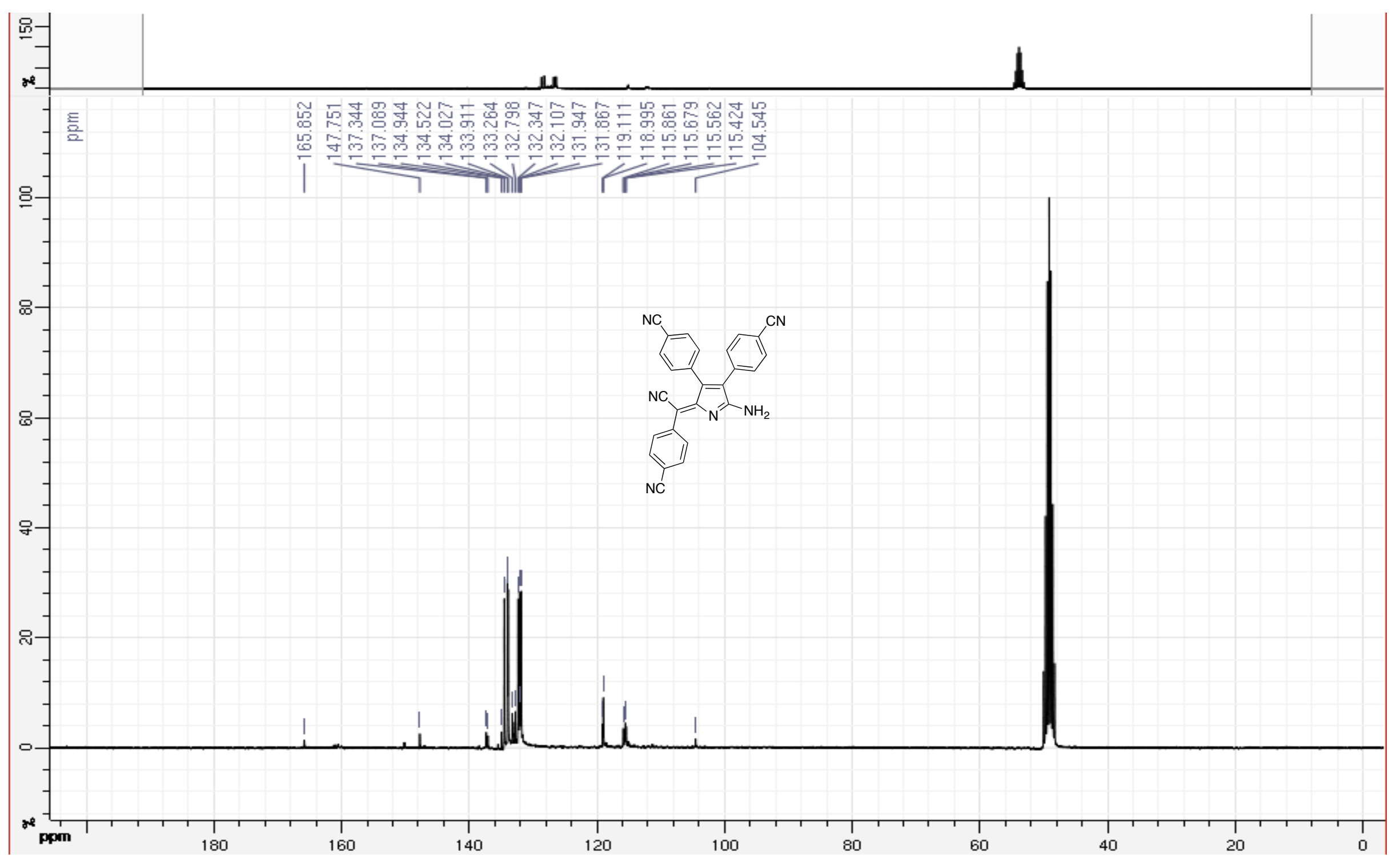


(E)-2-(5-Amino-3,4-di(pyridin-3-yl)-2H-pyrrol-2-ylidene)-2-(pyridin-3-yl)acetonitrile (2l)

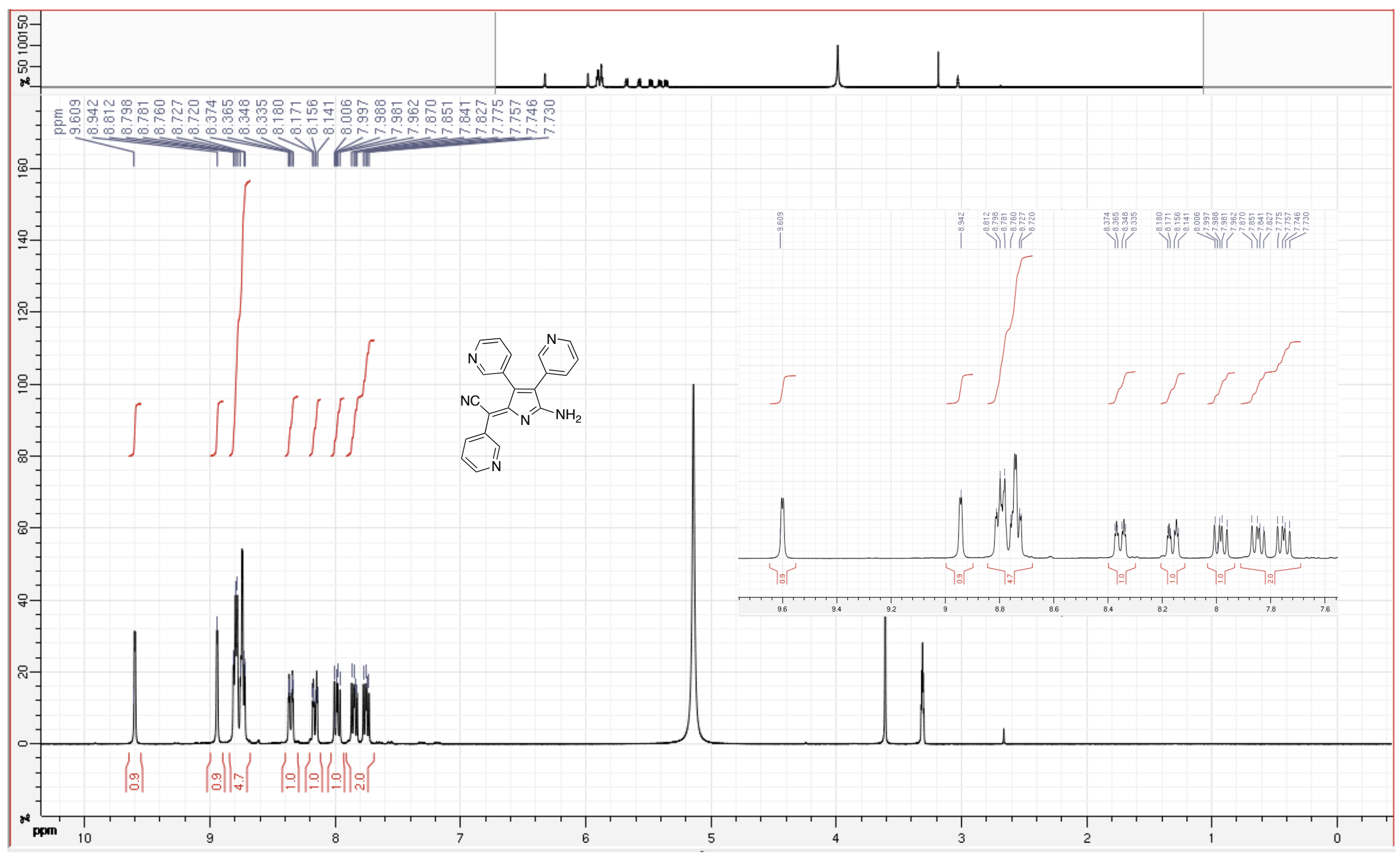




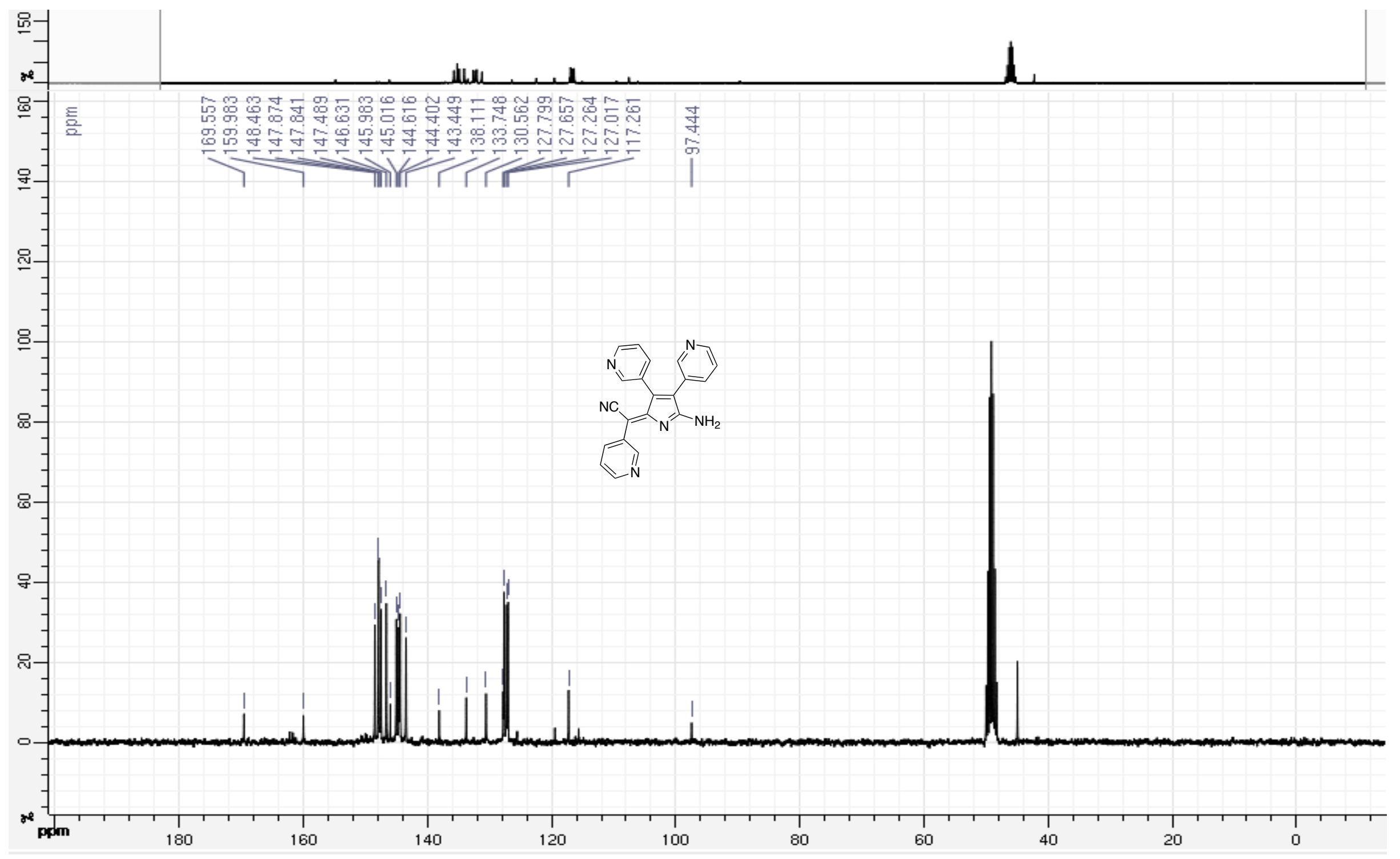


(Z)-2-(5-Amino-3,4-bis(4-isopropylphenyl)-2H-pyrrol-2-ylidene)-2-(4-isopropylphenyl)acetonitrile (2c')

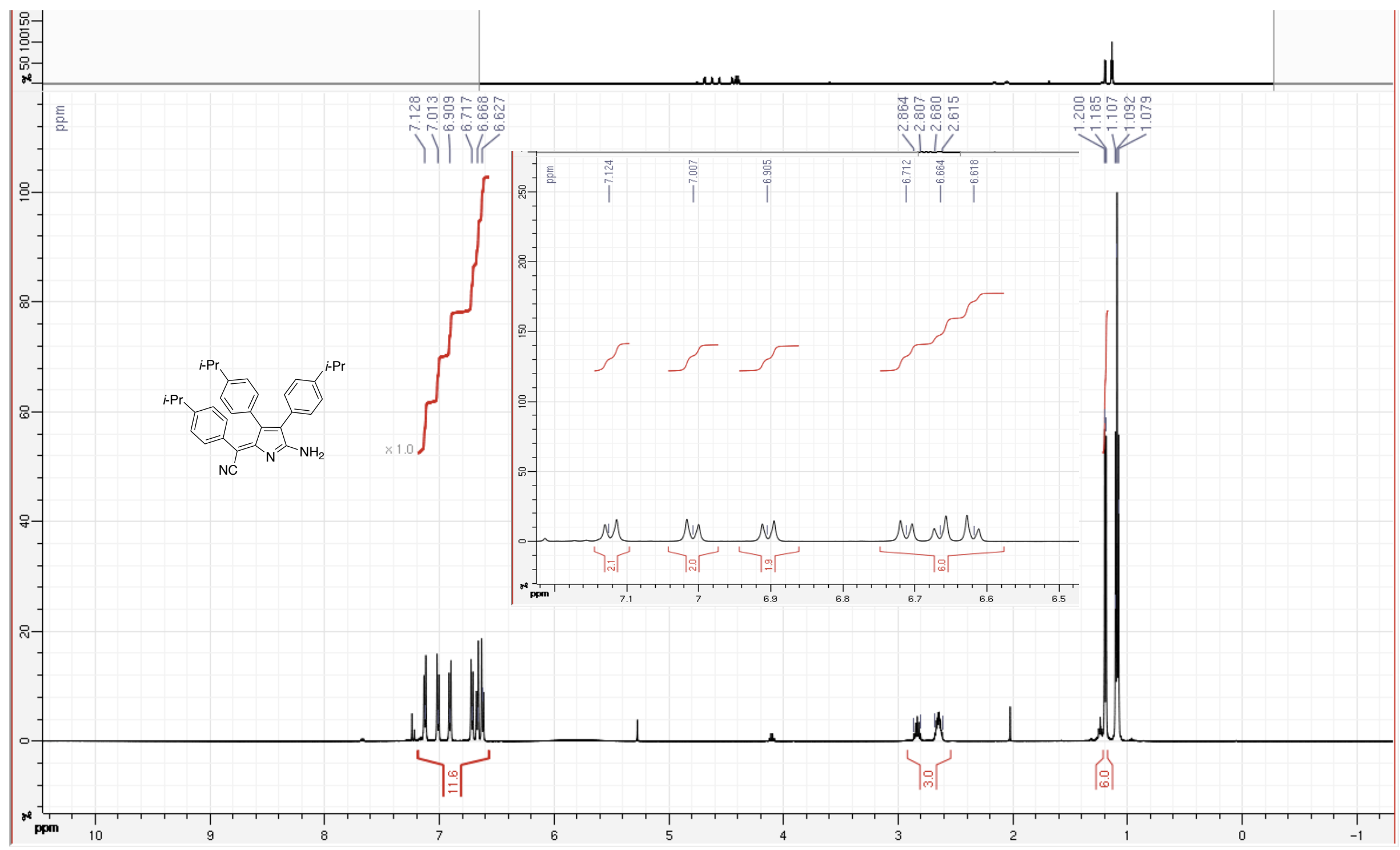




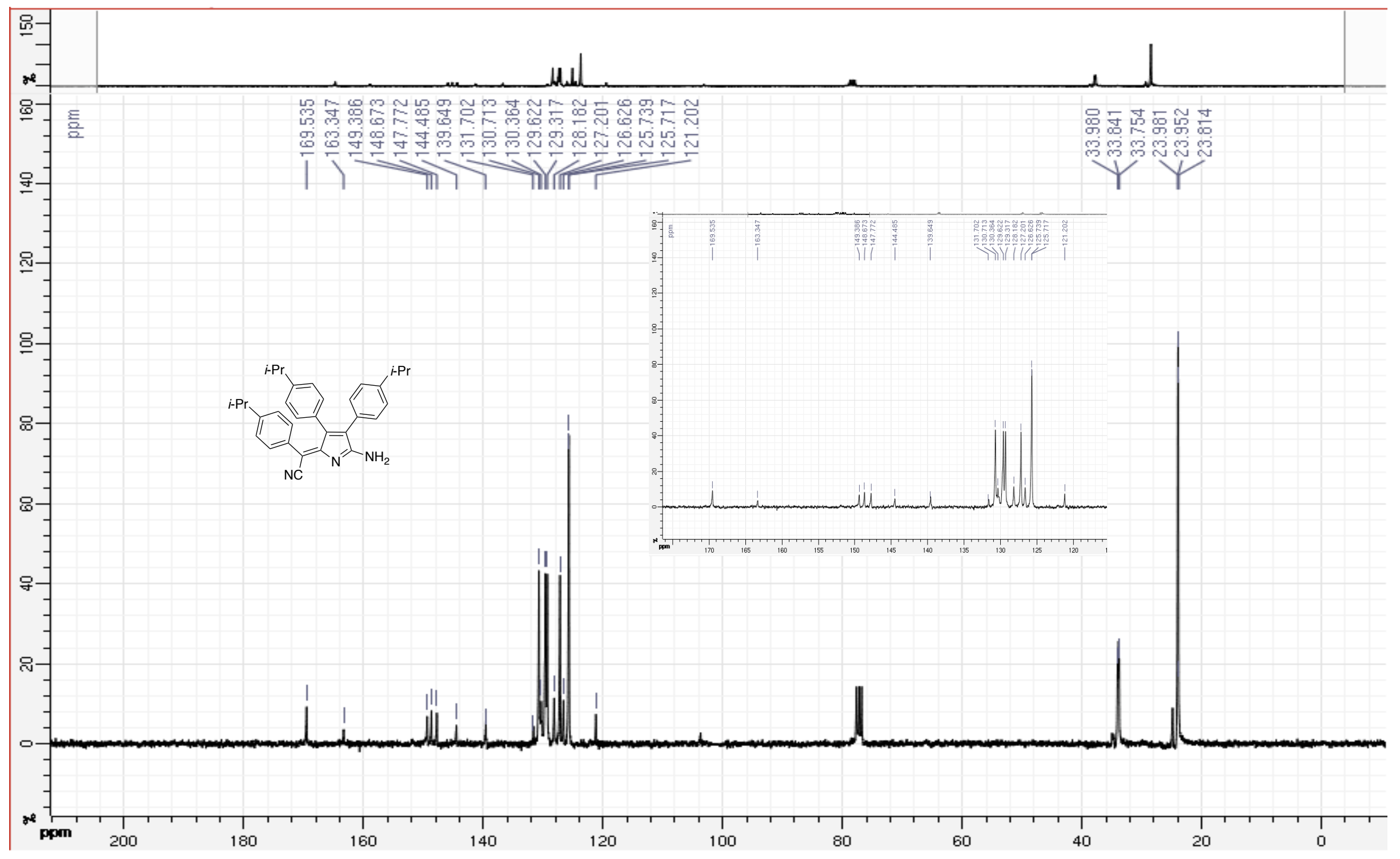

